\title{
IAEA CRP benchmark of ROCOM PTS test case for the use of CFD in reactor design using the CFD-Codes ANSYS CFX and TrioCFD
}

\author{
Thomas Höhne $^{\mathrm{a}, *}$, Sören Kliem ${ }^{\mathrm{a}}$, Ulrich Bieder ${ }^{\mathrm{b}}$

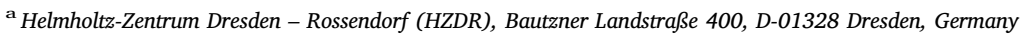 \\ ${ }^{\mathrm{b}}$ DEN-STMF, CEA, Université Paris-Saclay, F-91191 Gif-sur-Yvette, France
}

\begin{abstract}
A B S T R A C T
Over the last 15 years, considerable effort has been expended in assembling the available information on the use of CFD in the nuclear reactor safety field. Typical application areas here are heterogeneous mixing and heat transfer in complex geometries, buoyancy-induced natural and mixed convection, etc., with specific reference to Nuclear Reactor Safety (NRS) accident scenarios such as Pressurized Thermal Shock (PTS), boron dilution, hydrogen build-up in containments, thermal fatigue and thermal striping issues, etc. The development, verification and validation of CFD codes in respect to Nuclear Power Plant (NPP) design necessitates further work on the complex physical modelling processes involved, and on the development of efficient numerical schemes needed to solve the basic equations. Therefore, a set of ROCOM CFD-grade test data were made available to set up an International Atomic Energy Agency (IAEA) benchmark, relating to PTS scenarios. The benchmark deals with the injection of the relatively cold Emergency Core Cooling (ECC) water, which can induce buoyancydriven stratification. Data obtained from the PTS experiment were compared in the study presented here with predictions obtained from the CFD software packages ANSYS CFX and TrioCFD. In addition a test case without buoyancy forces was selected to show the influence of density differences.

The results of the two test cases and the numerical calculations show that mixing efficiency is strongly influenced by buoyancy effects. At higher mass flow rates without density differences the injected slug propagates in the circumferential direction around the core barrel. Buoyancy forces reduce this azimuthal propagation. The ECC water falls in an almost vertical path and reaches the lower downcomer sensor below the inlet nozzle. Therefore, density effects play an important role during natural convection with ECC injection in PWRs. Both CFD codes were able to predict the observed flow patterns and mixing phenomena.
\end{abstract}

\section{Introduction}

The last decade has seen an increasing use of three-dimensional CFD codes to predict steady state and transient flows in nuclear reactors because a number of important phenomena such as pressurized thermal shocks, coolant mixing, and thermal striping cannot be predicted by traditional one-dimensional system codes with the required accuracy and spatial resolution. The nuclear industry now also recognizes that CFD codes have reached the desired level of maturity (at least for single-phase applications) for them to be used as part of the NPP design process, and it is the objective of a Coordinated Research Project (CRP): Application of Computational Fluid Dynamics Codes for Nuclear Power Plant Design of the Department of Nuclear Energy Department of the International Atomic Energy Agency (IAEA) to assess the current capabilities of such codes in this regard, and contribute to the technology advance in respect to their verification and validation. CFD is already well-established in addressing certain safety issues in NPPs, as reported and discussed at various international workshops. The development, verification and validation of CFD codes in respect to NPP design necessitates further work on the complex physical modelling processes involved, and on the development of efficient numerical schemes needed to solve the basic equations. In parallel, it remains an overriding necessity to benchmark the performance of the CFD codes, and for this experimental databases need to be established, both for separate-effect tests and for full-size integral tests.

The IAEA has long been aware that there will be increasing interest in the use of CFD codes, and in particular in their verification, validation and uncertainty quantification, and joined with the OECD/NEA in sponsoring the initial exploratory drive to document the progress of CFD as a simulation tool in the field of nuclear reactor safety, and advance it by proposing numerical benchmarking exercises, and organizing international workshops. These jointly sponsored activities

\footnotetext{
* Corresponding author.

E-mail addresses: t.hoehne@hzdr.de (T. Höhne), ulrich.bieder@cea.fr (U. Bieder).
} 


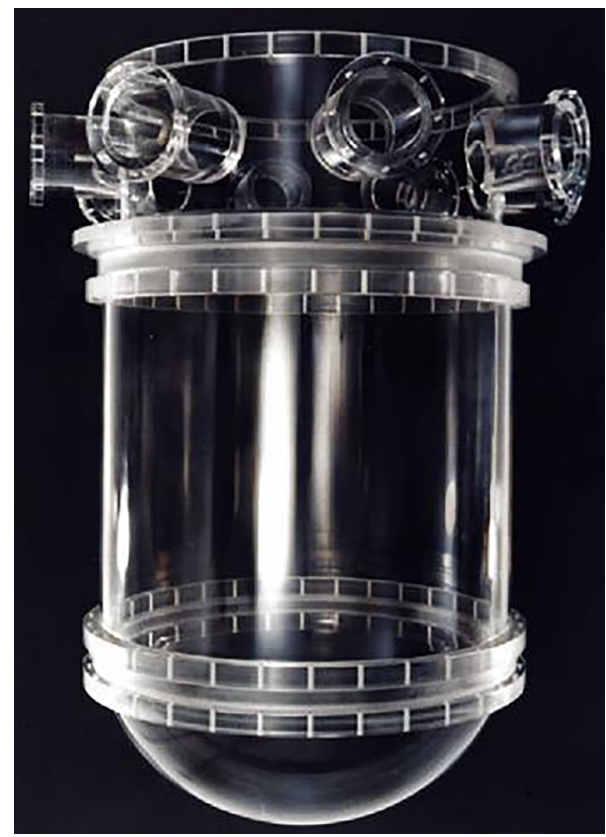

Fig. 1. The reactor model manufactured from Plexiglas( .

remain ongoing. The present CRP seeks to fill a void in the original initiative in recognition of the growing use of CFD tools in reactor design, while maintaining the existing synergy with continuing efforts in the reactor safety area with the NEA.

Therefore at the IAEA it was decided to set up a benchmark of two

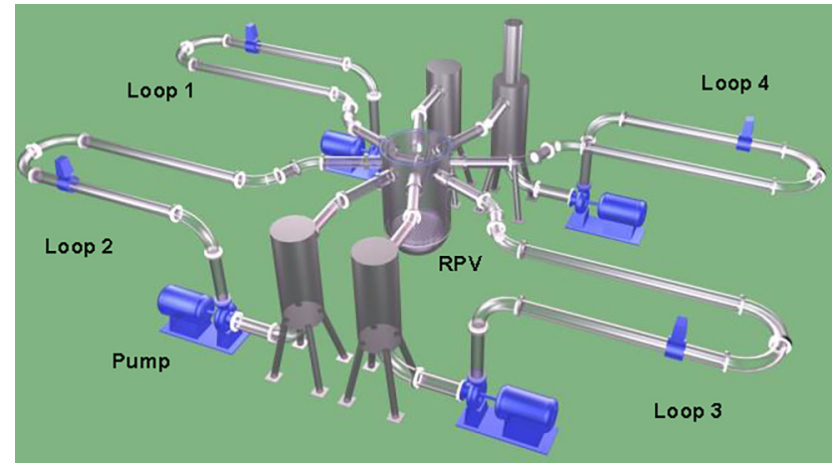

Fig. 2. ROCOM test facility with four loops and individually frequency controlled circulation pumps.

ROCOM CFD-grade experiments, see Höhne and Kliem (2016). The ROCOM facility is at 1:5 scaled mock-up, based on the 4-loop Konvoi reactor concept. There are 1000 measuring points by means of the Wire-Mesh Sensor (WMS) measurement technique, for which data collection is available up to a frequency of $10 \mathrm{kHz}$. All the test data had previously been opened, so only an 'open' benchmark exercise could be contemplated.

A set of ROCOM test data could be made available, relating to PTS and Boron dilution scenarios. It was noted that each experiment had been repeated five times to ensure authenticity of the data. In both cases, initial and boundary conditions are specified. Data have been recorded at P1 (95\%) and P2 (67\%) confidence levels. A CAD file of the test geometry is also available - it has recently been updated. All test data are available in tabular form, for ease of interpretation.

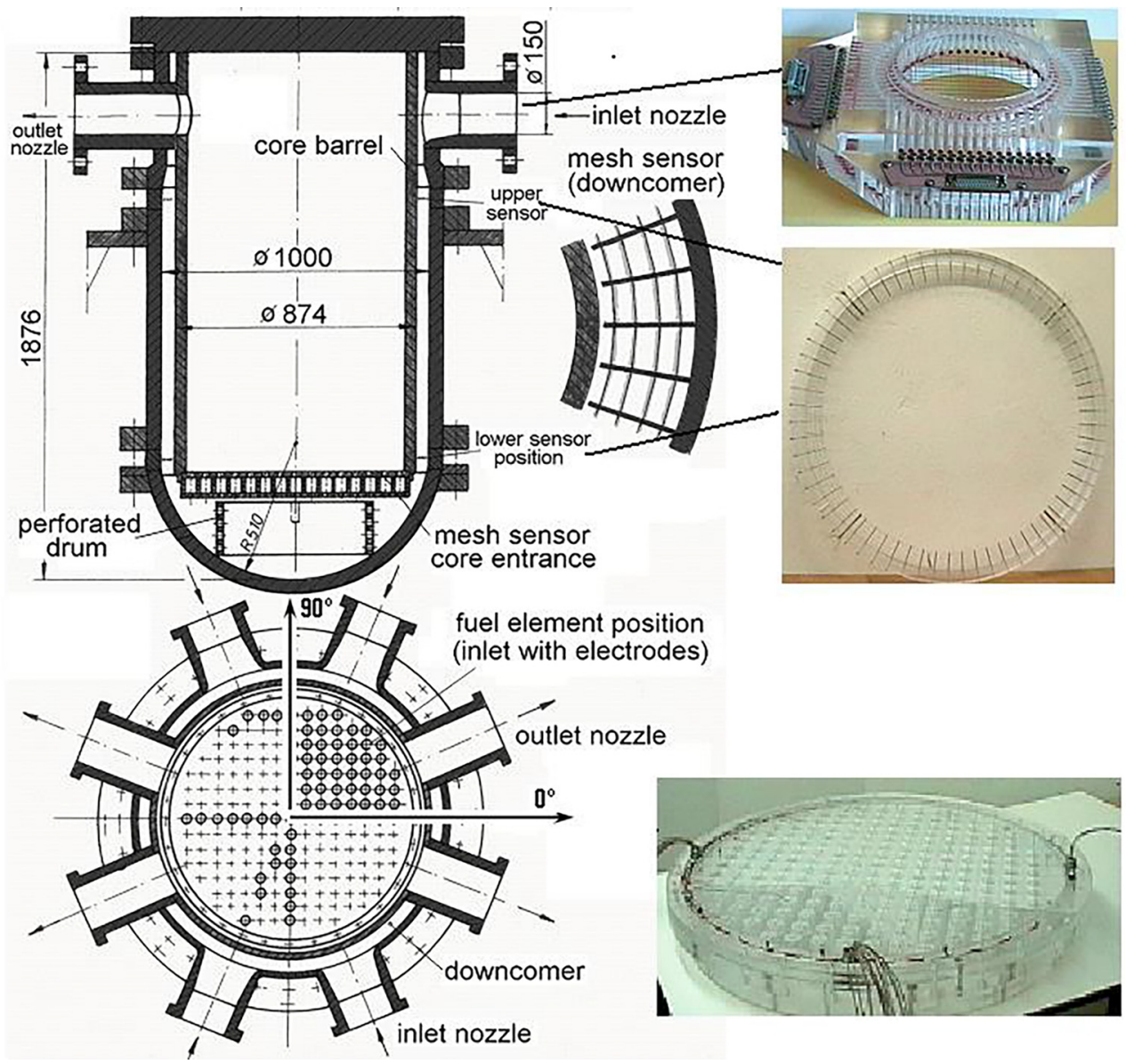

Fig. 3. Mesh sensor for measuring tracer distributions in front of the reactor inlet nozzle. 


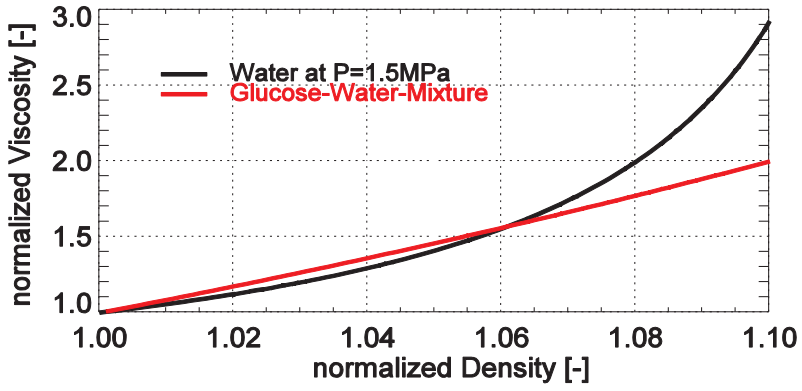

Fig. 4. Dependency of the normalized viscosity on the normalized density for water and glucose-water mixture.

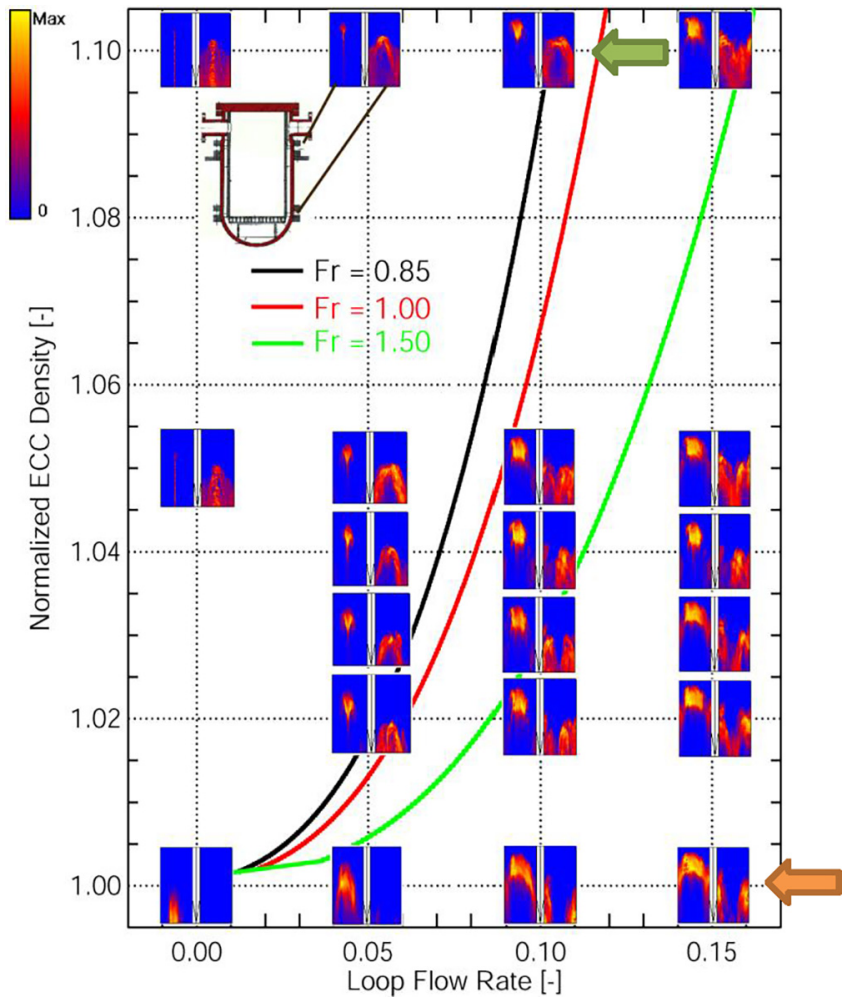

Fig. 5. Test matrix of ECC injection experiments. Visualization of the timedependent tracer distribution at the sensors in the downcomer; isolines of constant Froude numbers.

Table 1

Experimental runs.

\begin{tabular}{lllll} 
& $\begin{array}{l}\dot{V}(\mathrm{ECC})\left[\mathrm{m}^{3} /\right. \\
\mathrm{h}]\end{array}$ & $\begin{array}{l}\dot{V}\left(\mathrm{loop} \mathrm{n}^{\circ} 1\right) \\
{\left[\mathrm{m}^{3} / \mathrm{h}\right]}\end{array}$ & $\begin{array}{l}\rho_{\mathrm{ECC}} / \rho_{\text {loop }} \text { ECC } \\
\text { water }: \text { loop }{ }^{\circ} 1\end{array}$ & $\begin{array}{l}\text { Fr in the } \\
\text { downcomer }\end{array}$ \\
\hline $\mathrm{d} 10 \mathrm{~m} 10$ & 3.6 & 18.5 & $1100: 1000$ & 0.82 \\
$\mathrm{~d} 00 \mathrm{~m} 15$ & 3.6 & 27.75 & $1000: 1000$ & $\infty$ \\
\hline
\end{tabular}

The benchmark experiments analyzed in this paper are devoted to PTS scenarios. Density differences between the coolant water and the primary loop inventory can play an important role during loss-ofcoolant accidents in nuclear power plants, as the injection of the relatively cold ECC water can induce buoyancy-driven stratification. This stratification can cause high temperature gradients and increased thermal stresses of the reactor pressure vessel. Moreover, in the case of inadvertent injection of ECC water with low boron concentration, a boron dilution transient could be initiated, leading to unstable core operation. Such issues are of concern to the safety of the NPP, but also need to be addressed at the design stage, since they have a direct influence on the projected life-time of the plant.

This paper shows results of post-test numerical simulations of the PTS benchmark experiment. Experimental data obtained for a constant flow rate in one loop, which represents the magnitude of natural circulation, and $0 \%$ (called d00m15) respectively $10 \%$ (called $\mathrm{d} 10 \mathrm{~m} 10$ ) density difference between ECC and loop water were compared with numerical predictions from the CFD codes CFX and TrioCFD. The first comparison of these codes on ROCOM mixing experiments have been made in 2005 and published by Höhne et al. (2006) for a pure buoyancy driven flow (d10m5). Since then CEA and HZDR have developed and validated standardized strategies to analyze flow mixing in the RPV by CFD calculations, which are based on best practice guideline procedures. These strategies are applied in this paper on a mixed convection and a forced convection experiment, which have not been analyzed by CFD before.

\section{ROCOM test facility}

The Mixing Test Facility ROCOM (Prasser et al., 2003; Kliem et al., 2008) consists of a reactor pressure vessel model (Fig. 1) with four inlet and four outlet nozzles. The Facility is equipped with four fully operating loops (Fig. 2), i.e. it has four circulation pumps, which are driven by motors with computer controlled frequency transformers. As a result, a wide variety of flow rate regimes, such as four-loop operation, operation with pumps off, simulated natural circulation modes and flow rate ramps can be realized. Beginning from the bends in the cold legs, which are closest to the reactor inlet, the geometrical similarity between model and original reactor is respected until the core inlet. The core itself is excluded from the similarity; a core simulator is used to keep the Euler-number constant (similar pressure drop model/prototype). The reactor model is manufactured from Plexiglas@ (Fig. 1).

A total view of the test facility is given in Fig. 2. The reactor model describes the geometry of the original PWR with respect to the design of the nozzles (diameter, curving radii and diffuser parts), the characteristic extension of the downcomer cross section below the nozzle zone, the so-called perforated drum in the lower plenum and the design of the core support plate with the orifices for the coolant. The flow rate in the loops is scaled according to the transit time of the coolant through the reactor model. Since the geometrical scale of the facility is $1: 5$, the transition time of the coolant is identical to the original reactor, when the coolant flow rate is scaled down by $1: 125$. The nominal flow rate in ROCOM is therefore $185 \mathrm{~m}^{3} / \mathrm{h}$ per loop. The coolant in the disturbed loop was labeled by injecting a sodium chloride solution into the main coolant flow in front of the affected reactor inlet nozzle. Magnetic valves controlled start and stop of the injection process.

\section{Instrumentation}

The tracer distribution in the reactor model was observed by socalled electrode-mesh sensors, which measure the distribution of the electrical conductivity over the cross section of the flow duct. The own development was aimed at a direct conductivity measurement between pairs of crossing wires to avoid tomographic reconstruction algorithms and to reach a time resolution of up to 10000 frames per second (Prasser et al. 1998). Measured local conductivities are afterwards related to reference values. The result is a so-called mixing scalar that characterizes the instantaneous share of coolant originating from the disturbed loop (i.e. where the tracer is injected) at a given location inside the flow field. The scalar is dimensionless. Assuming similarity between tracer field and the temperature and boron concentration fields under fully turbulent flow conditions it can be used to apply the experimental results to the original reactor. The reference values correspond to the unaffected coolant (index 0 ) and the coolant at the disturbed reactor inlet nozzle (index 1 ). The difference between the two 


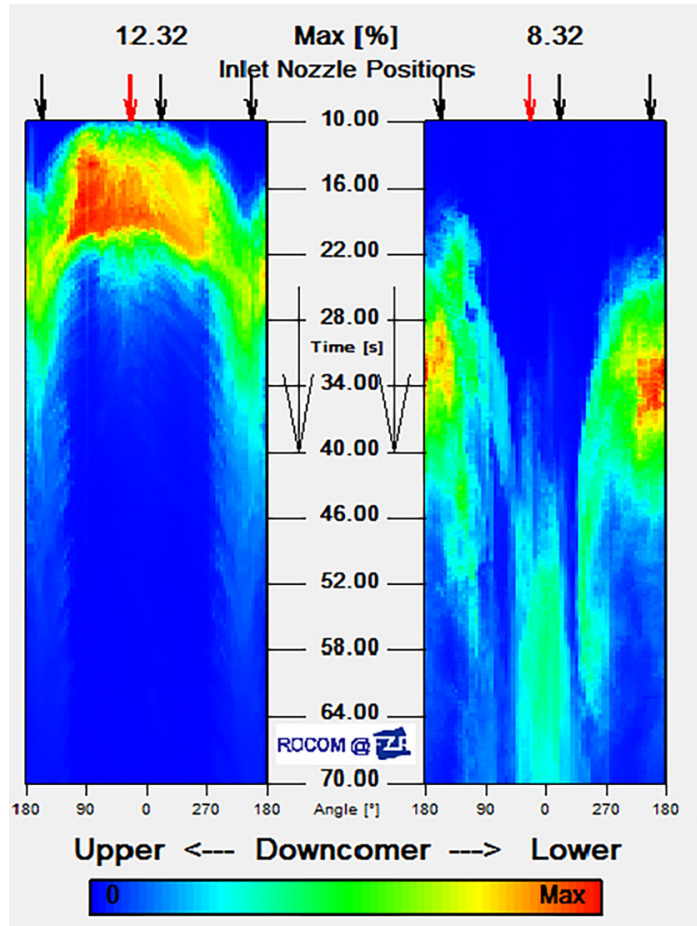

do0m 15

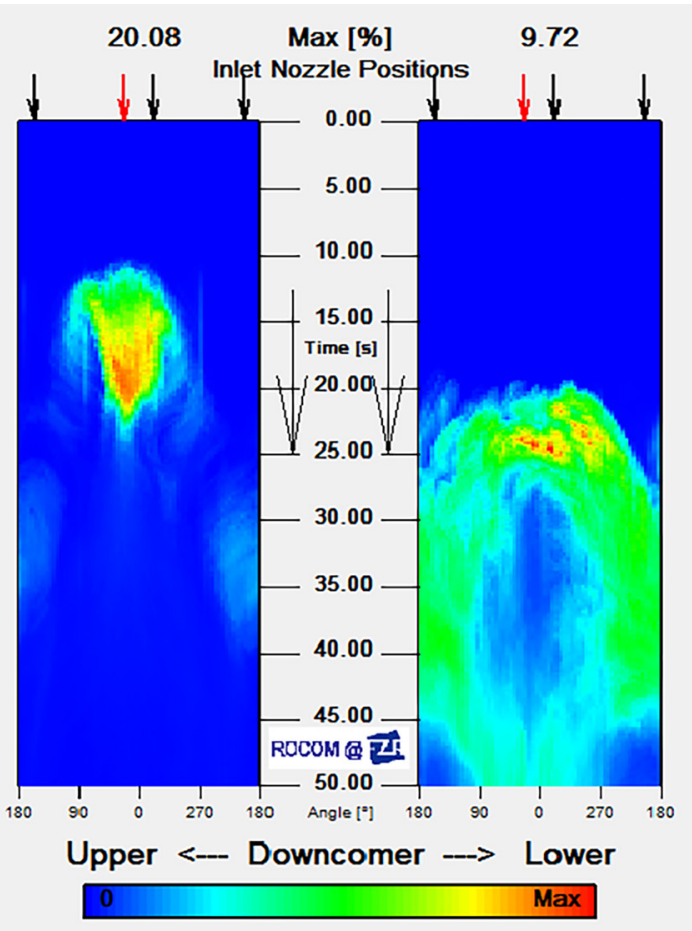

d10m10

Fig. 6. Time dependent tracer distributions at the Upper and Lower Downcomer Sensor.

Table 2

Solver for the CFD calculations setup.

\begin{tabular}{lll}
\hline Advection scheme & Option & High Resolution \\
\hline \multirow{2}{*}{ Transient scheme } & $\Delta \mathrm{t}$ & 2nd Order Backward Euler \\
& & $0.05 \mathrm{~s}$ \\
Convergence control & Timescale control & Inner iterations \\
& Min./max. coeff. loops & min. 4/max. 50 \\
Convergence criteria & Residual type & RMS \\
& Residual target & $10^{-4}$
\end{tabular}

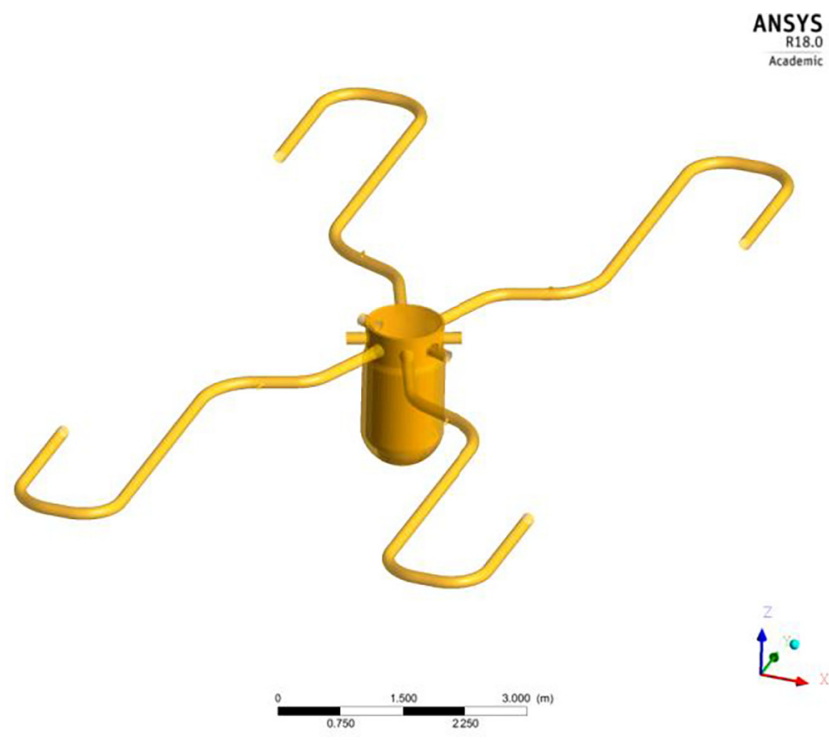

Fig. 7. Flow domain with RPV and 4 cold legs (perspective view).

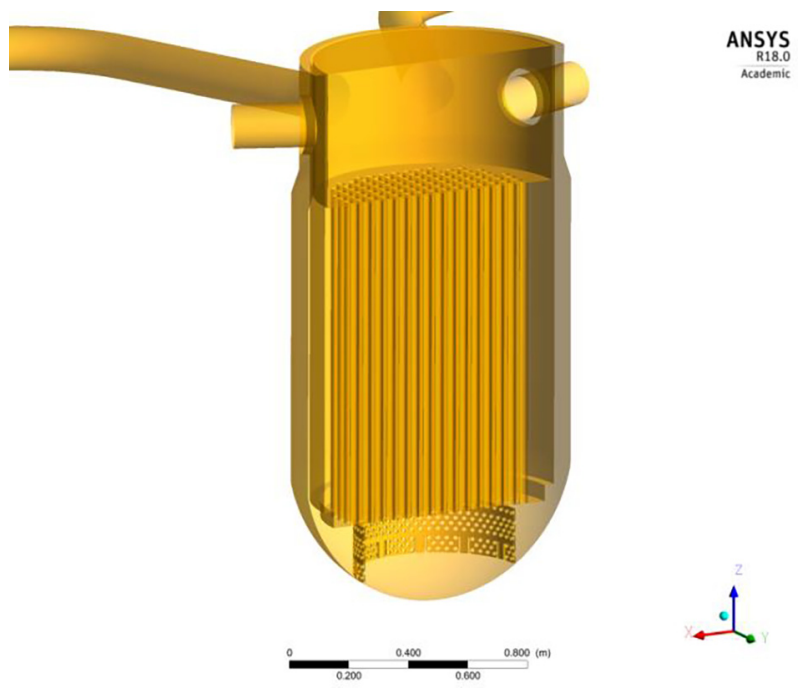

Fig. 8. View on the internal structures (vertical cut).

reference values is the magnitude of the perturbation. The mixing scalar $\Theta$ is defined as follows in Eq. (1):

$\theta_{x, y, z, t}=\frac{\sigma_{x, y, z, t}-\sigma_{0}}{\sigma_{1}-\sigma_{0}} \cong \frac{T_{x, y, z, t}-T_{0}}{T_{1}-T_{0}} \cong \frac{C_{B, x, y, z, t}-C_{B, 0}}{C_{B, 1}-C_{B, 0}}$

Here, $\sigma$ is the electrical conductivity, $T$ is the temperature and $C_{B}$ the boron concentration. Which of the two parameters temperature or boron concentration is represented by the measured mixing scalar depends only on the right choice of the reference values and the set-up of the boundary conditions in the experiment. The conductivity is linked to the salt concentration, salt is added to the ECC, and it is assumed that the same mixing between sugar and salt is taken place. 


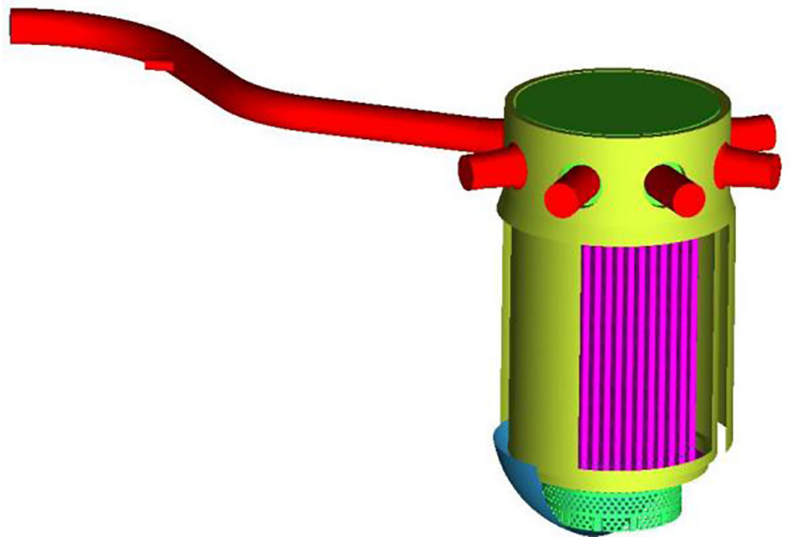

Fig. 9. Visualization of the CAD model.

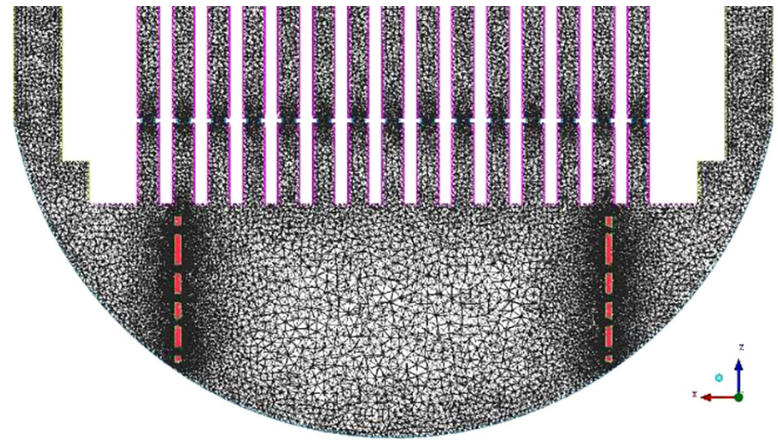

Fig. 10. Meshing in the lower plenum.

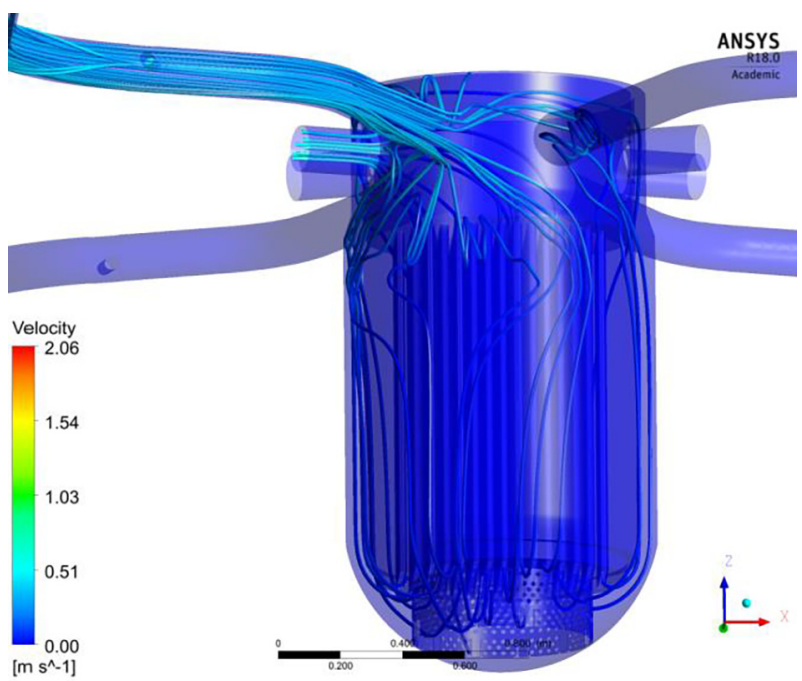

Fig. 11. Streamlines representing the fluid flow before the injection takes place (4 s), CFX-calculation.

Mesh sensors are placed at four positions of the flow path. The first sensor (Fig. 3) is flanged to the reactor inlet nozzle (Fig. 1) in loop $n^{\circ} 1$. It is aimed at the observation of the distribution at the reactor inlet. The second and the third sensors are located in the downcomer. The downcomer sensors consist of radial fixing rods with orifices for four circular electrode wires. The fourth sensor is integrated into the core support plate. $2 \times 15$ electrode wires are arranged in a way that the wires of the two planes cross in the centers of the coolant inlet orifices

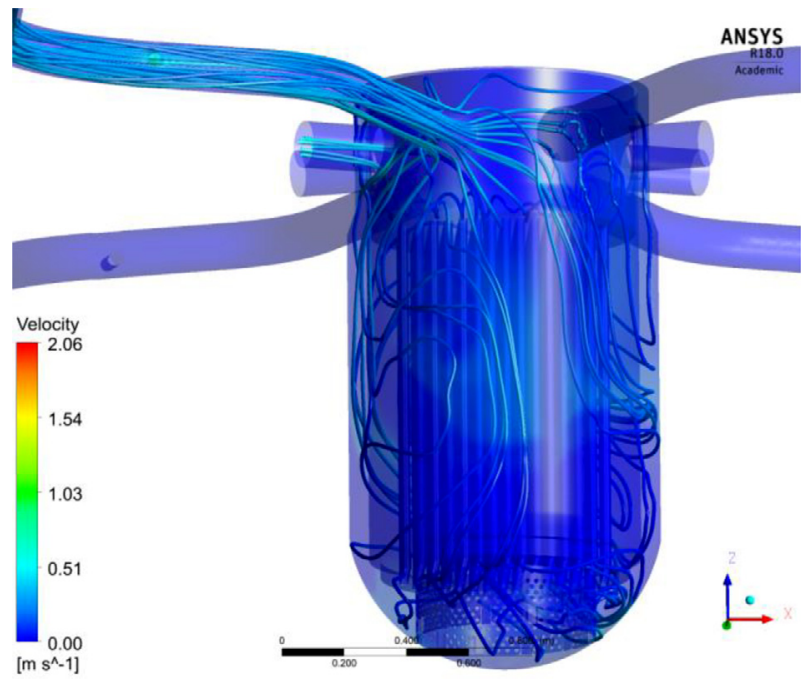

Fig. 12. Streamlines representing the fluid flow after the injection took place (23 s), CFX-calculation.
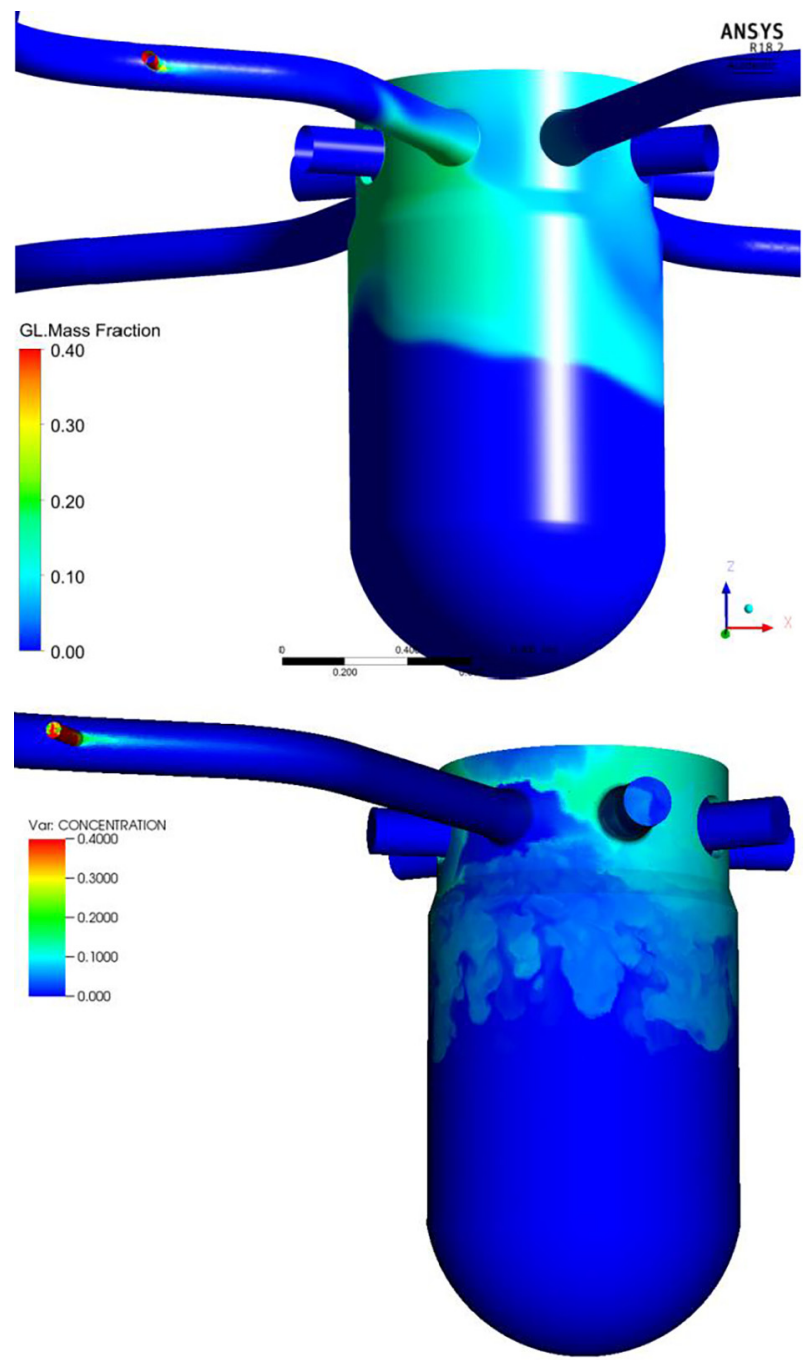

Fig. 13. Tracer distribution in the downcomer ( $18 \mathrm{~s}$ ), upper figure CFX, lower figure TrioCFD.

of each fuel element. In this way, the tracer concentration is measured at each fuel element inlet. In total, about 1000 measuring points are recorded, the measuring frequency is $200 \mathrm{~Hz}$. In most of the cases 10 


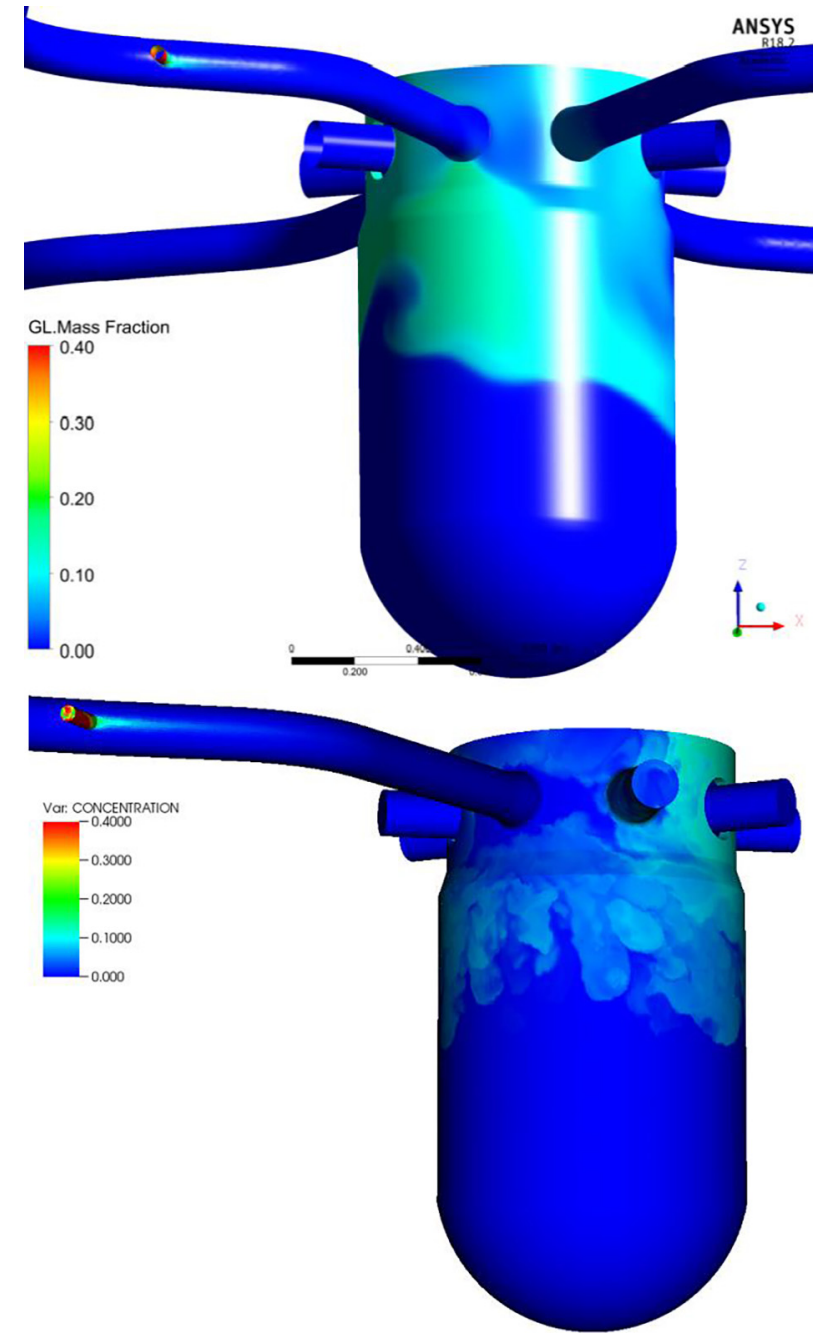

Fig. 14. Tracer distribution in the downcomer (20 s), upper figure CFX, lower figure TrioCFD.

successive measurements were averaged and the result was stored with a frequency of $20 \mathrm{~Hz}$, because the characteristic frequency of the observed phenomena was not requiring a higher sampling frequency.

\section{Pressurised thermal shock test case}

Density differences between the relatively cold ECC water injected during loss-of-coolant accidents in NPPs and the hot water in the primary loop inventory can play an important role in the safety of ageing NPPs by inducing potentially dangerous buoyancy-driven temperature stratifications. Such stratifications can give rise to severe temperature gradients, and thereby high associated thermal stresses in the reactor pressure vessel.

Data obtained for one particular experiment with an induced (constant) ECC flow rate in one of the four loops of magnitude identical with that of natural circulation, but in one case without density differences and in the other case with a density difference $10 \%$ higher than that of the loop water, has been chosen to form the basis of this numerical benchmarking exercise. As the ROCOM facility cannot be heated, the higher density of the cold ECC water is simulated by adding sugar (glucose). A sugar solution with a density of $1100 \mathrm{~kg} / \mathrm{m}^{3}$ has at a pressure of $1.5 \mathrm{MPa}$ a viscosity of about $50 \%$ below that of pure water (see Fig. 4). The sugar tracer can therefore still be regarded as a fluid with low viscosity.
The objective of the series of PTS experiments carried out in ROCOM was to investigate the effects of density differences between the primary loop inventory and the ECC injected water on the mixing in the RPV downcomer. The mass flow rate was varied between 0 and $15 \%$ of the nominal flow rate; i.e. it was kept at the same order of magnitude as that of the natural circulation mode. The density difference between the ECC water and the loop water was varied between 0 and 10\%. Fig. 5 displays pictorially the experimental results. In total, 21 experiments were carried out to form the test matrix (Höhne et al., 2009), as indicated by the individual images in Fig. 5. The experiments marked by the arrows are subject of the analysis presented here.

Analysis of all the experiments has been presented by Rohde et al. (2005) and Höhne et al. (2006). In their paper, the authors develop a criterion for the distinction between momentum-driven and densitydriven flows, based on the experimental data collected from these tests, and as determined by the densimetric Froude number Fr, defined in Eq. (2) in the usual way:

$F r=\sqrt{\frac{\rho v^{2}}{\Delta \rho g l}}$

From the entire set of data, the first experiment with $10 \%$ (constant) flow rate in one loop and 10\% density difference between ECC and loop water has been selected for the calculations to be performed during this benchmark exercise (see Table 1). In Table 1, the experiments are classified by the nomenclature $d x m y$, where $\mathrm{x}$ is the percentage of density difference and $y$ is the percentage of nominal mass flow rate in the cold leg. The Froude number for this test is $F r=0.85$, and may therefore be regarded as density-affected according to the evidence provided in Fig. 5 (present tests indicated by the arrows). This test represents the transition region between momentum driven and density driven flow. The second experiment in Table 1 and Fig. 5 has 15\% (constant) flow rate in one loop and $0 \%$ density difference between ECC and loop water. Fig. 6 visualizes in unwrapped views the time evolution of the tracer concentration measured at the two downcomer sensors for both experiments.

In all experiments, the volume flow rate of the ECC injection line was kept constant at $3.6 \mathrm{~m}^{3} / \mathrm{h}$. The normalized density ( $\rho_{\mathrm{ECC}} / \rho_{\text {loop }}$ ) is defined as the ratio between the density of water in the ECC line and density of fluid in the circuit. All other boundary conditions are identical. Due to the observed fluctuations of the flow field in the RPV, each experiment was repeated five times to average over these fluctuations. An error analysis showed that the experimental data have an error range of $\pm 3 \%$.

The experiments without density effects serve as reference experiments for the comparison. Fig. 6 (left case) visualizes in unwrapped views the time evolution of the tracer concentration measured at the two downcomer sensors. The downwards directed red arrow indicates the position of the loop with the running pump, in that case delivering $15 \%$ of the nominal flow rate. No density difference was created (d00m15). At the upper downcomer sensor, the ECC water appears directly below the inlet nozzle. Due to the momentum created by the pump, the flow entering the downcomer is divided into two streams flowing right and left in a downwards directed helix around the core barrel. At the opposite side of the downcomer, the two streaks of the flow merge together and move down through the measuring plane of the lower downcomer sensor into the lower plenum. Such a flow distribution is typical for single-loop operation. It is dominated by the momentum insertion due to the operating pump or high natural circulation flow rate. The maximum tracer concentration of the ECC water in the downcomer is $12 \%$ of the injected water concentration at the upper sensor and $8 \%$ at the lower sensor.

Fig. 6 (right case) shows the experiment d10m10, carried out at $10 \%$ of the nominal flow rate, but the density difference between the injected ECC water and the primary loop coolant is now $10 \%$. In that case the ECC water spreads less in azimuthal direction and forms a wide 


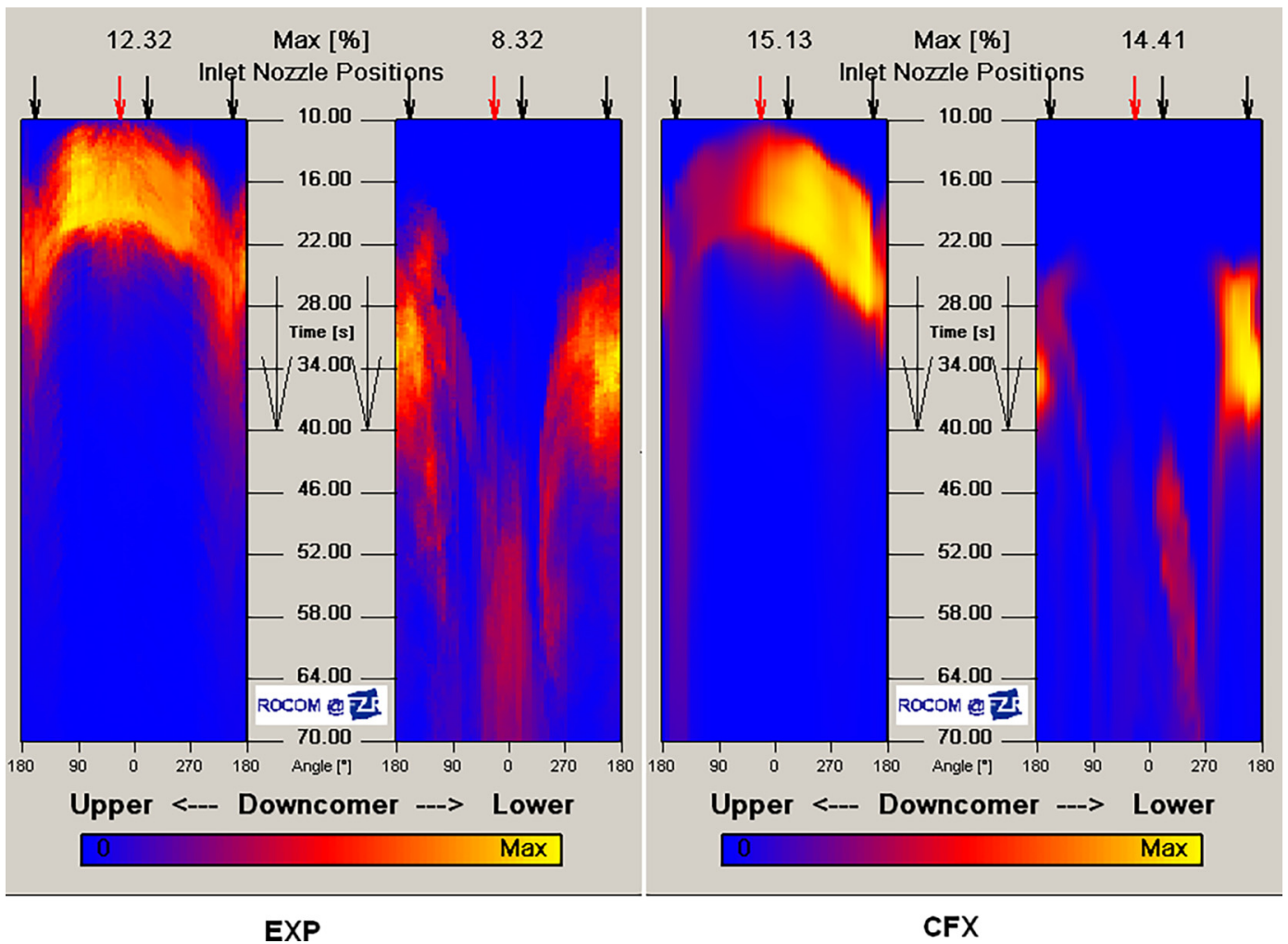

Fig. 15. Time dependent tracer distributions at the Upper and Lower Downcomer Sensor, left hand side experiment, right hand side CFX.

streak of water with higher density flowing down the downcomer. Hence, the ECC water covers a much smaller azimuthal sector at the upper sensor. The density difference partly suppresses the propagation of the ECC water in horizontal direction. The ECC water flows down in an almost straight streamline and reaches the lower downcomer sensor below the affected inlet nozzle. Later, coolant containing ECC water appears also at the opposite side of the downcomer.

The visualizations of the different behavior of ECC water in the downcomer reveals that in the case of momentum driven flow, the ECC water covers nearly the whole perimeter of the upper sensor and passes the measuring plane of the lower sensor mainly at the opposite side of the downcomer. When density effects are present, the sector at the upper measuring device covered by the ECC water is smaller. The ECC water flows down straightly and passes the sensor in the lower part of the downcomer below the inlet nozzle of the working loop.

\section{Numerical modelling with ANSYS CFX}

One CFD code for simulating the mixing studies was ANSYS CFX (Ansys CFX, 2017). The discretization in space is a 2nd order elementbased finite-volume method with 2nd order time integration. It uses a coupled algebraic multigrid algorithm to solve the linear systems arising from discretization. The discretization schemes and the multigrid solver are scalably parallelized. CFX works with unstructured hybrid grids consisting of tetrahedral, hexahedral, prism and pyramid elements.

\subsection{Numerical scheme, nodalization, time step size and turbulence modelling}

The overall error of a CFD calculation is a combination of several aspects: Grid density, discretization method, time step size, iteration error and the employed mathematical models have all their own effect. The separation of these error components for complex three- dimensional calculation is difficult. For example discretization errors can act like an additional numerical diffusivity, and can affect the results in a similar way as a too large eddy viscosity arising from an unsuitable turbulence model. Discretization errors can be reduced by using finer grids, higher-order discretization methods and smaller time step sizes. However, in many practical three-dimensional applications grid- and time step-independent solutions cannot be obtained because of hardware limitations. In these cases, the remaining errors and uncertainties should be quantified as described in the OECD Best Practice Guidelines (BPG) by Mahaffy et al. (2007). The glucose water, which had a higher density, was modelled with the multi-component model of CFX. In a multi-component flow, the components share the same velocity, pressure and temperature fields. The properties of multi-component fluids are calculated on the assumption that the constituent components form an ideal mixture. The glucose water is modelled as a component with different density and viscosity compared to water. The mass fraction of the glucose water can be directly related to the mixing scalar described in Eq. (1).

The reference fluid is demineralized water at ambient conditions ( 1 bar and $20^{\circ} \mathrm{C}$ ). The density differences are artificially produced with added sugar, there are no temperature differences in the test rig. The amount of the tracer $\mathrm{NaCl}$ has no additional influence on density, the viscosity of the sugar water of $24 \%$ mass saccharose $(10 \%$ higher density) has 4 times higher viscosity than demineralized water at ambient conditions. The Reynolds stress model (RSM) proposed by Launder et al. (1975), which is based on the Reynolds Averaged NavierStokes Equations (RANS), was used in combination with an $\omega$-based length scale equation (BSL model) to model the effects of turbulence on the mean flow. Buoyancy production terms were included in the Reynolds stress equations and in the length-scale equation. The buoyancy production terms in the Reynolds stress equations are exact terms and require no modelling.

The simulations were run on the HZDR Linux-Cluster "Hydra" with the solver setup in Table 2. It consists of two heads and 72 compute 


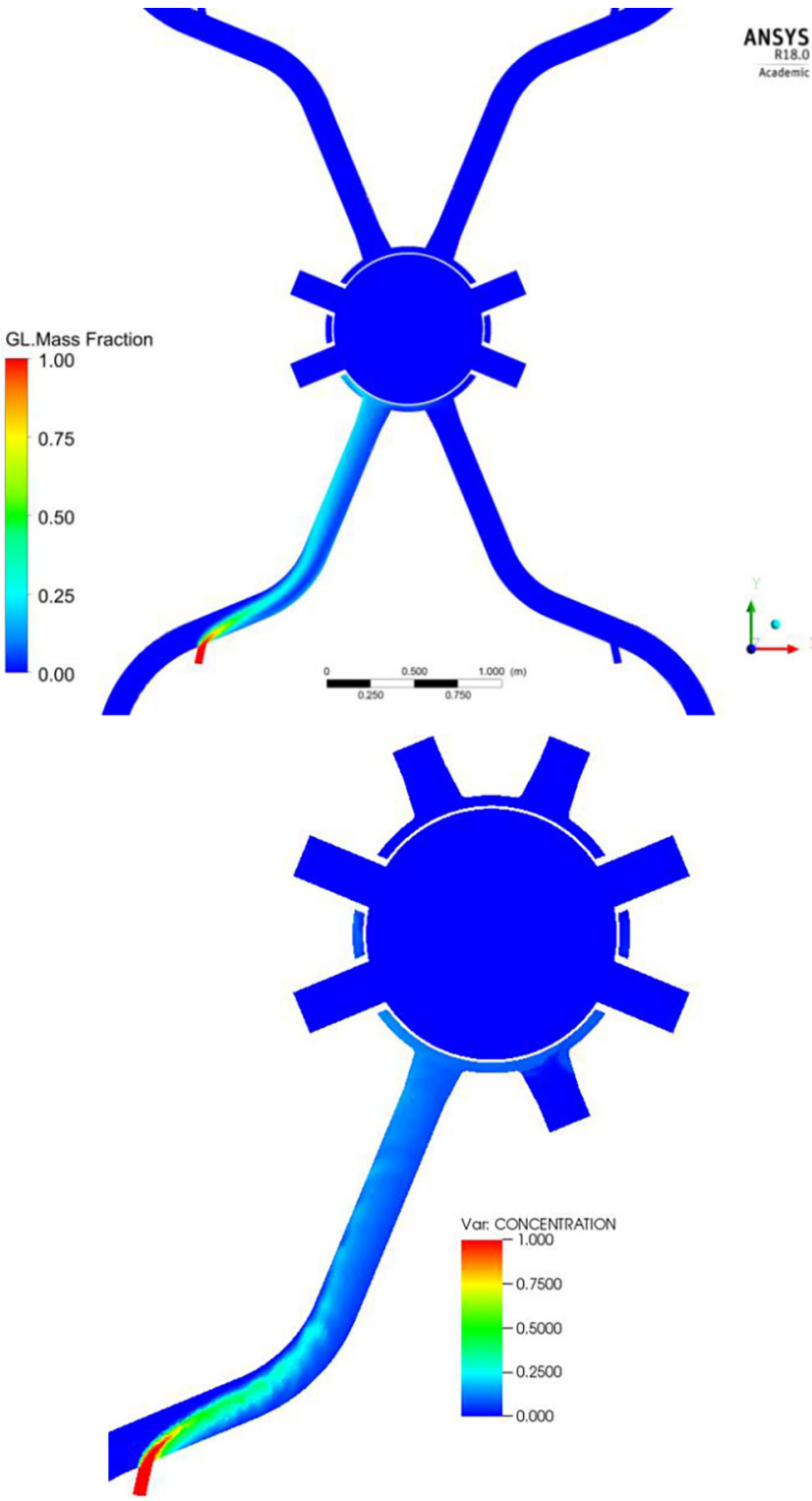

Fig. 16. Tracer distribution in the cold leg (10 s), upper figure CFX, lower figure TrioCFD.

nodes. Each node has 2 Intel CPUs (Xeon 8-Core or 16-Core). In general hydra consists of more than $1500 \mathrm{CPU}$-cores and $8.5 \mathrm{~TB}$ of main memory. The network is a $1 \mathrm{GbE}$ Ethernet and additionally an InfiniBand fabric with a bandwidth of $40 \mathrm{Gbit} / \mathrm{s}$, which qualifies hydra for sequential, coarse granularity parallel and massively parallel jobs. The operating system is Ubuntu Linux. The theoretical general peak performance (single precision) amounts to $70.5 \mathrm{Tflop} / \mathrm{s}$. The calculation of $50 \mathrm{~s}$ real time took $23 \mathrm{~h}$ on 32 processor nodes.

\subsection{Geometrical Simplifications, local details}

The geometric details of the ROCOM internals have a strong influence on the flow field and on the mixing. Therefore, an exact representation of the inlet region, the downcomer below the inlet region, and the obstruction of the flow by the outlet nozzles in the downcomer is necessary (see Figs. 7 and 8). In the current study, these geometric details were modelled using the ICEMCFD software (Ansys ICEMCFD, 2017). The final model included the inlet nozzles with the diffuser part, the orifices of the outlet nozzles, the downcomer extension, the lower plenum, the core support plate, the perforated drum, the core

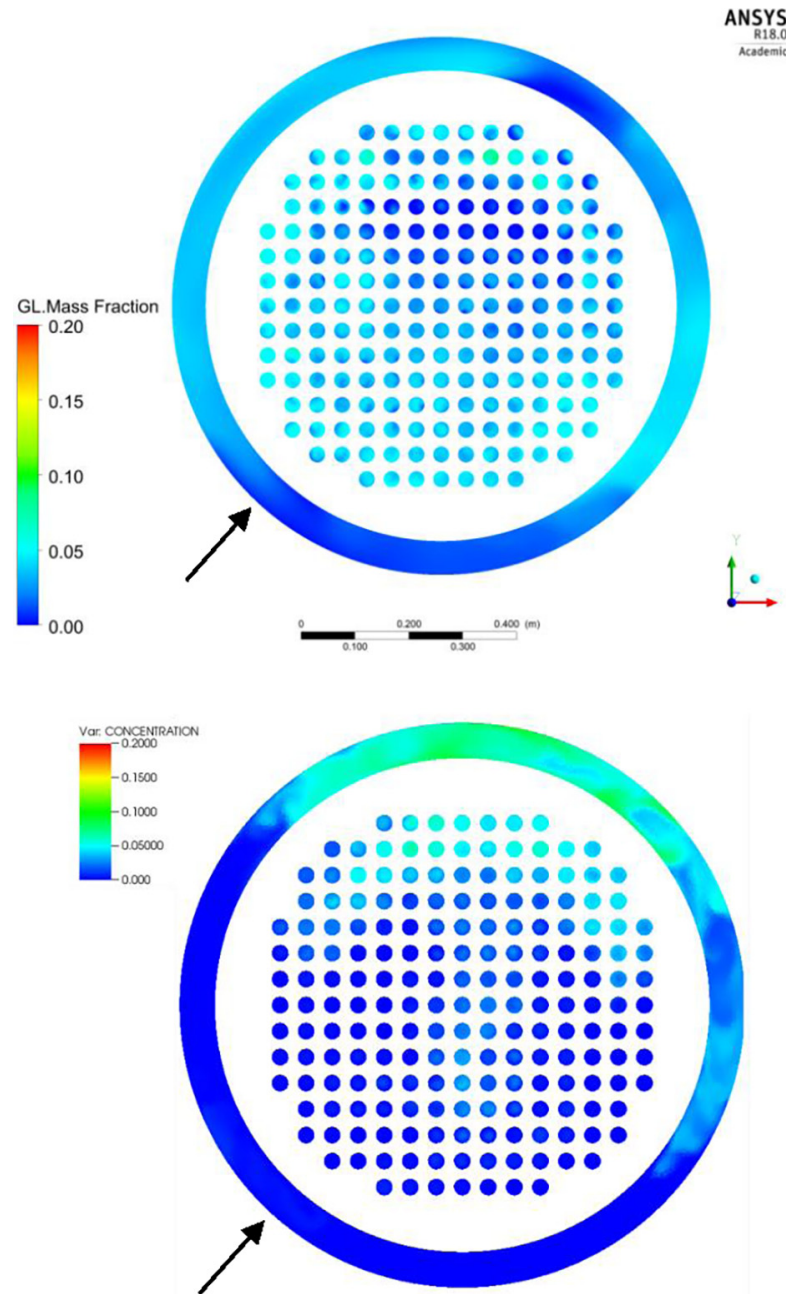

Fig. 17. Tracer distribution in the core inlet ( $34 \mathrm{~s}$ ), upper figure CFX, lower figure TrioCFD.

simulator, the upper plenum, and the outlet nozzles. As an example, the core support plate contains 193 orifices with a diameter of $20 \mathrm{~mm}$. Then the fluid flows through the hydraulic core simulator inside the tubes. The perforated drum, shown in Fig. 8, contains 410 orifices of $15 \mathrm{~mm}$ diameter.

\subsection{Grid generation}

The mesh was generated with the ICEMCFD (Ansys ICEMCFD, 2017) software. It consisted of 6.5 million nodes and about 4 million hybrid elements for the total calculation domain shown in Fig. 7. The mesh was refined at the following reactor pressure vessel internals shown in Fig. 8: perforated drum, lower support plate and ECC injection line. The downcomer and nozzle region was discretized with hexahedral cells; tetrahedral elements were used for the lower plenum.

\section{Numerical modelling with TrioCFD}

TrioCFD is the CFD reference code of the Nuclear Energy Division of the CEA (Angeli et al., 2015). The code is developed at CEA-Saclay and was renamed in 2015 from Trio_U to TrioCFD.

\subsection{Numerical scheme, nodalization, time step size and turbulence modelling}

TrioCFD uses a finite volume based finite element approach on 


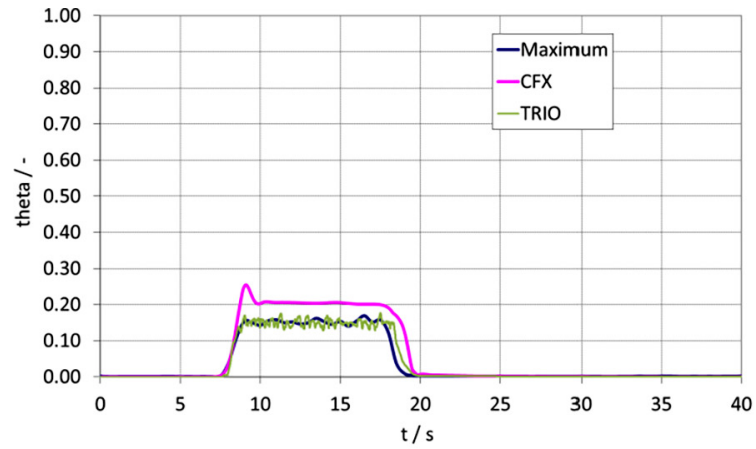

Maximum Mixing Scalar

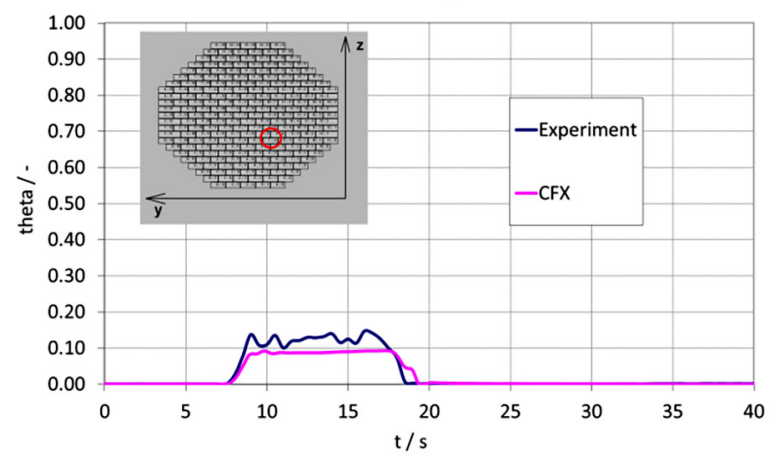

Local Mixing Scalar Pos. 0704

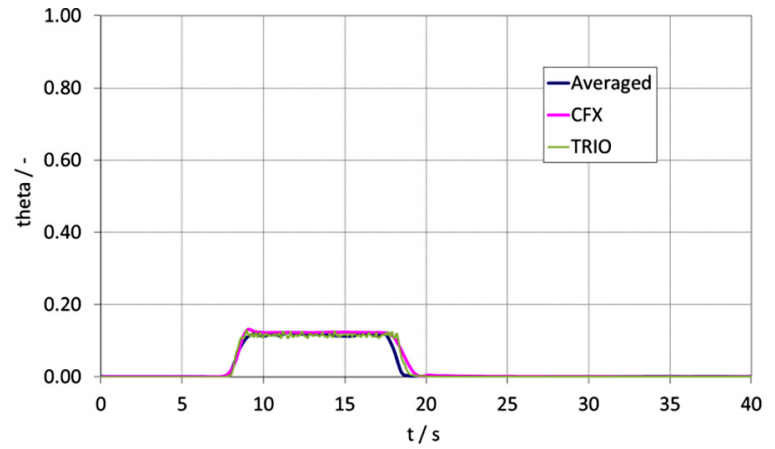

Average Mixing Scalar

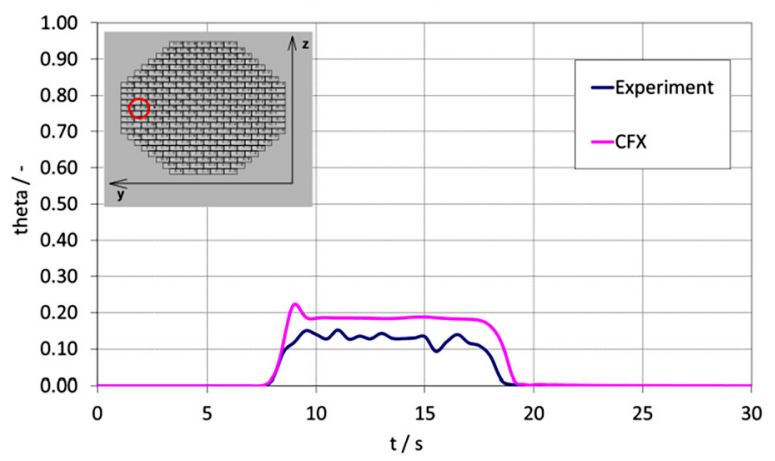

Local Mixing Scalar Pos. 0312

Fig. 18. Time dependent tracer course at the cold leg inlet nozzle sensor.

tetrahedral cells to integrate in conservative form all conservation equations over the control volumes belonging to the calculation domain. As in the classical Crouzieux-Raviart element (Crouzieux and Raviart, 1973), both vector and scalar quantities are located in the center of the faces. The pressure, however, is located in the vertices and the center of gravity of a tetrahedral element as shown by Angeli et al. (2017). This discretization leads to a very good pressure/velocity coupling. The 2nd order precise explicit Adams-Bashforth scheme is used for time integration. The used time step respects the Courant-Friedrichs-Levy stability criteria of CFL $<0.8$. The convection scheme for scalars of Kuzmin and Turek (2004), was generalized to vectors leading to a slightly stabilized 2nd order precise centered convection scheme as described by Ducros et al. (2010). The diffusion term is discretized by a 2nd order centered scheme. In the calculations presented here, density variations are taken into account by the Boussinesq approximation. The density is thus taken constant in all terms of the Navier-Stokes equations except in the gravity term where the dependency of the density on the mixing scalar concentration is linearized according to the GlucoseWater Mixture shown in Fig. 4. The dynamic viscosity is calculated as a function of the saccharose concentration as shown in Fig. 4. Turbulence is treated by large eddy simulations (LES). The WALE model (Nicoud and Ducros, 1999) is used to account for the sub grid scale dissipation. The discretized momentum conservation equations are integrated following the SOLA pressure projection method of Hirt et al. (1975). The resulting Poisson equation is solved with a conjugate gradient method using a symmetric successive over relaxation technique to improve convergence. The convergence threshold at each time step has been set to $10^{-7}$, what leads during the transient to a very good conservation of the mass.

The presented LES calculations have been carried out exploiting the parallel calculation capabilities of the code. Each domain is decomposed into several overlapping sub-domains by using METIS libraries; all sub-domains were equally distributed among different processor cores using message passing interface libraries (MPI), which communicate mutually only when data transfer is needed.

\subsection{Geometrical simplifications and meshing}

The CAD model of the CFX calculations (Fig. 7) was simplified by reducing the length of the closed cold legs as shown in Fig. 9. Cold leg $\mathrm{n}^{\circ} 1$, ECC water injection line, downcomer, perforated drum and core simulant are identical to the CFX calculation. The meshing was realized with ICEMCFD (Ansys ICEMCFD, 2017). A meshing with 22 million tetrahedral elements was created by using the Delaunay method. At least 10 calculation points are located in the holes of the perforated drum (Fig. 10).

The $60 \mathrm{~s}$ real time of the experiment has been simulated with a mean time step of about $0.0008 \mathrm{~s}$ (except during the ECC inject phase). The transient has been calculated in about $30 \mathrm{~h}$ on 1008 processor nodes of the HPC machine Curie of the Très Grand Centre de Calcul of CEA. Curie is a BULL cluster with a peak power of $2 \mathrm{Pflop} / \mathrm{s}$. This power is reached by 11,520 Intel Nehalem and Intel Sandy Bridge processor nodes, representing 92,160 computing cores with a memory of 360 terabytes.

\section{Boundary conditions}

The analyzed experiments were described in Table 1 . For the experiment $\mathrm{d} 00 \mathrm{~m} 15$, the main pump $\mathrm{n}^{\circ} 1$ is delivering $15 \%$ of the nominal mass flow rate. The density difference between the injected ECC water and the primary loop coolant is $0 \%$. The other pumps are not working. For the experiment $\mathrm{d} 10 \mathrm{~m} 10$, the main pump $\mathrm{n}^{\circ} 1$ is delivering $10 \%$ of the nominal mass flow rate and the density difference is $10 \%$. Also the other pumps are not working. For both codes and both experiments, Dirichlet inlet boundary conditions were specified at the ECC injection line and after the bend of the cold leg $n^{\circ} 1$. Constant, uniform velocities were used to simulate the one-loop operation. During a period of $10 \mathrm{~s}$, a uniform velocity profile was also defined at the ECC injection line. At all other times, this velocity is set to zero. The mass fraction of glucose water was set to $1(\mathrm{~d} 10 \mathrm{~m} 10)$ and set to 0 at $\mathrm{d} 00 \mathrm{~m} 15$ at the ECC injection line and to 0 at cold leg $n^{\circ} 1$. For the CFX calculations, the same 

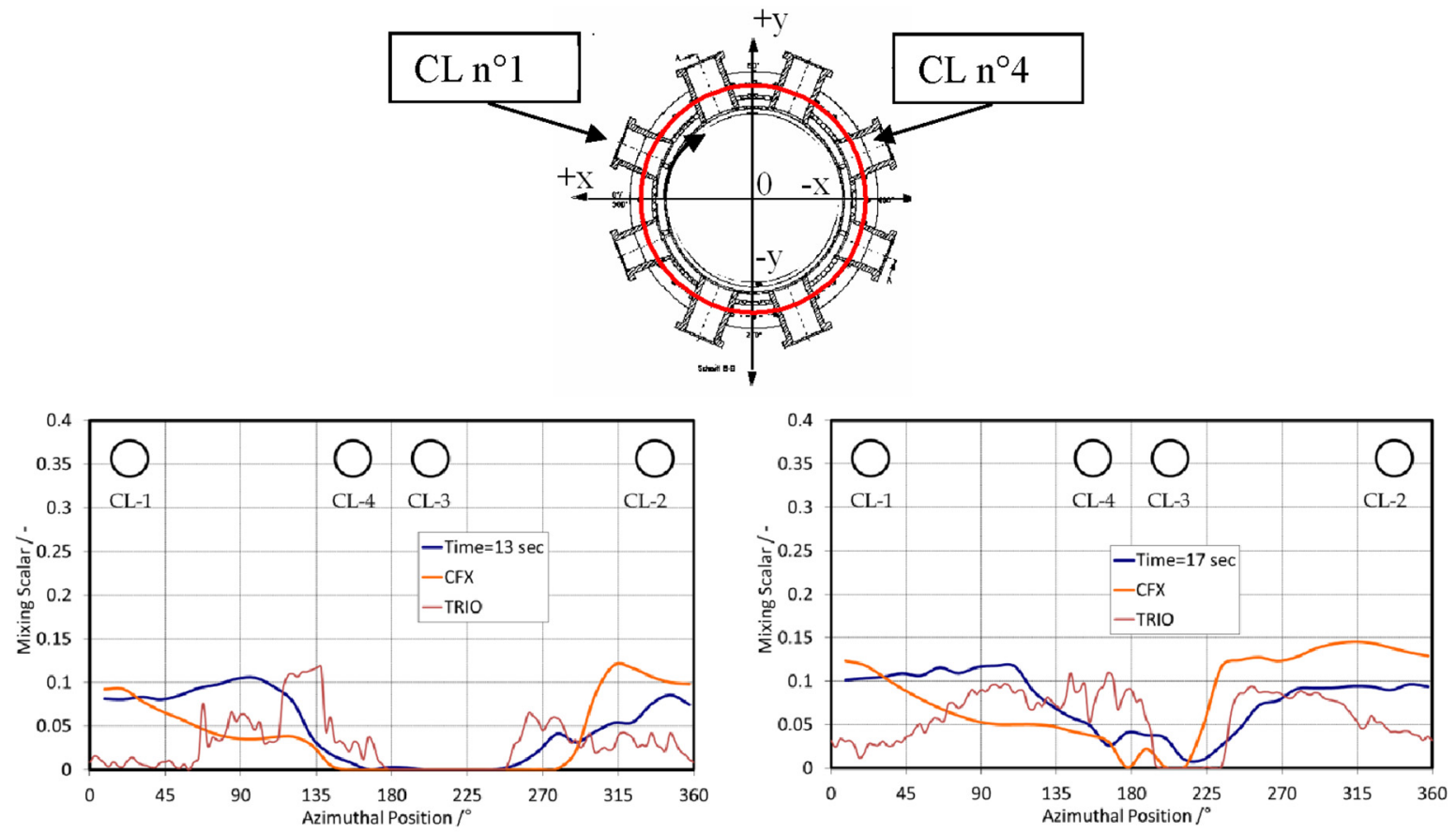

Upper downcomer Sensor
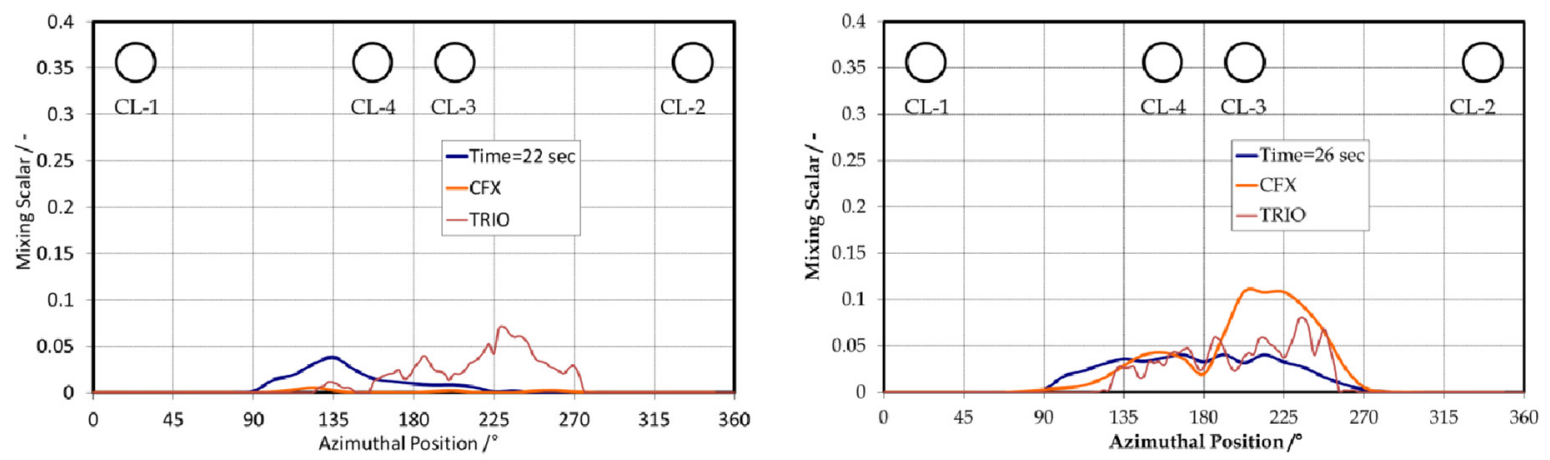

Lower downcomer Sensor

Fig. 19. Instantaneous circumferential tracer distributions for the 32 positions in the center of the upper and lower downcomer sensor.

constant static pressure was specified at all outlet nozzles. A no-slip boundary condition with automatic linear/logarithmic wall functions was used at all solid walls. In TrioCFD, von Neumann conditions with the same imposed pressure are applied at all hot leg outflow faces. The general wall law of Reichardt (1951) is used to model momentum exchange between walls and fluid; this law spans with one correlation the three layers of viscous-, buffer- and logarithmic law region.

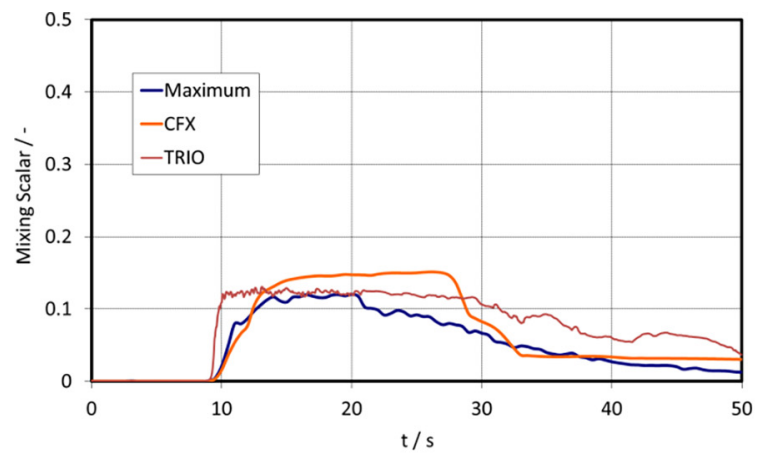

Maximum Mixing Scalar

\section{Initial conditions}

In both calculations the initial velocity in loop $\mathrm{n}^{\circ} 1$ was set to $10 \%$ and $15 \%$, respectively, of the nominal flow $\left(18.5 \mathrm{~m}^{3} / \mathrm{h}\right.$ for $\mathrm{d} 10 \mathrm{~m} 10$ and $27.75 \mathrm{~m}^{3} / \mathrm{h}$ for $\left.\mathrm{d} 00 \mathrm{~m} 15\right)$. As the pumps of the idle loops were not operating, these loops were closed and the velocities were defined as zero. In order to better initialize the velocity field in the vessel, the flow in

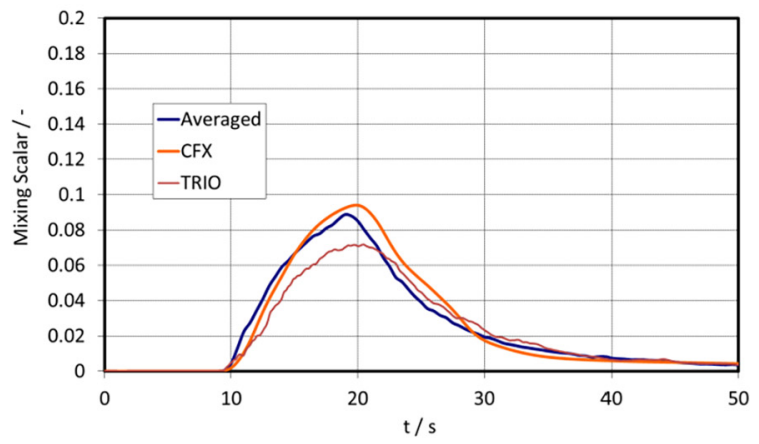

Averaged Mixing Scalar

Fig. 20. Time dependent tracer distributions at the upper downcomer sensor. 

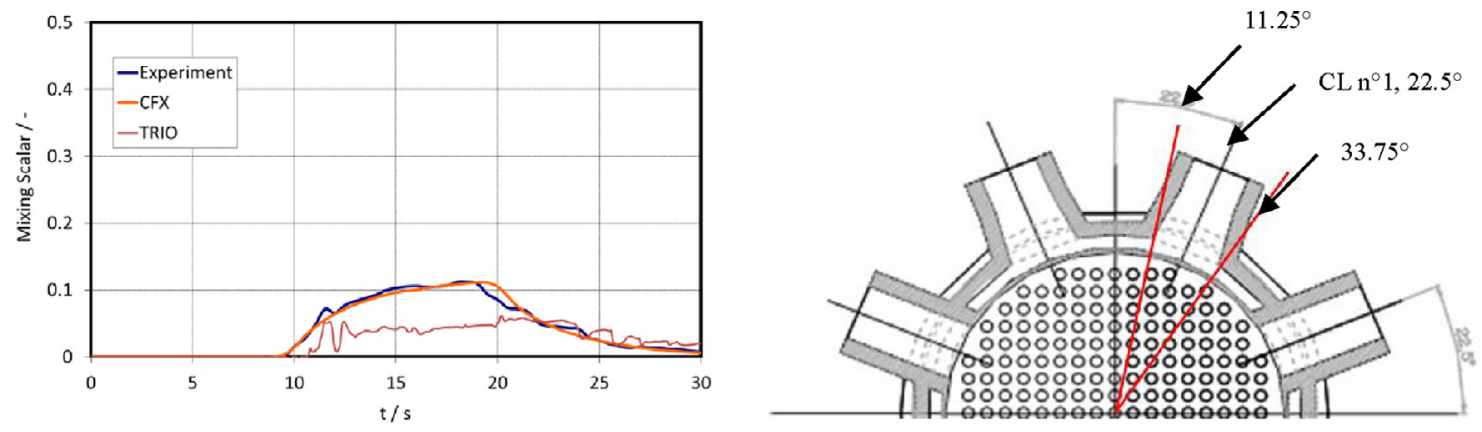

Local Mixing Scalar Pos. 22.5

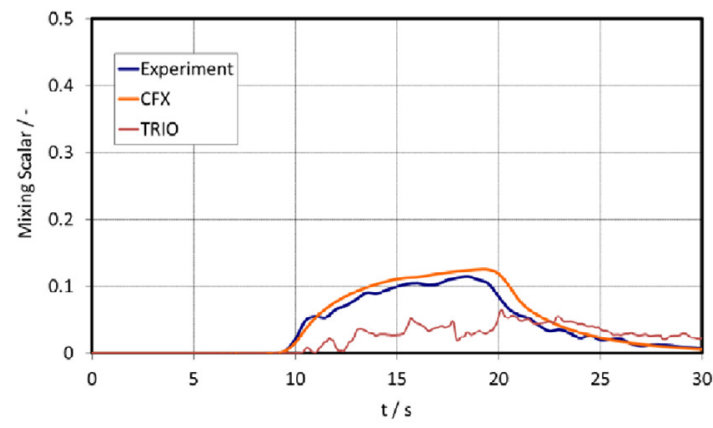

Local Mixing Scalar Pos. $11.25^{\circ}$

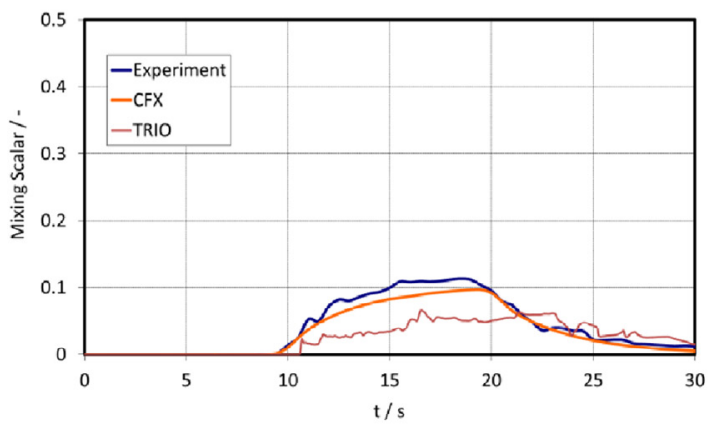

Local Mixing Scalar Pos. $33.75^{\circ}$

Fig. 21. Local transient tracer distributions at the upper downcomer sensor.

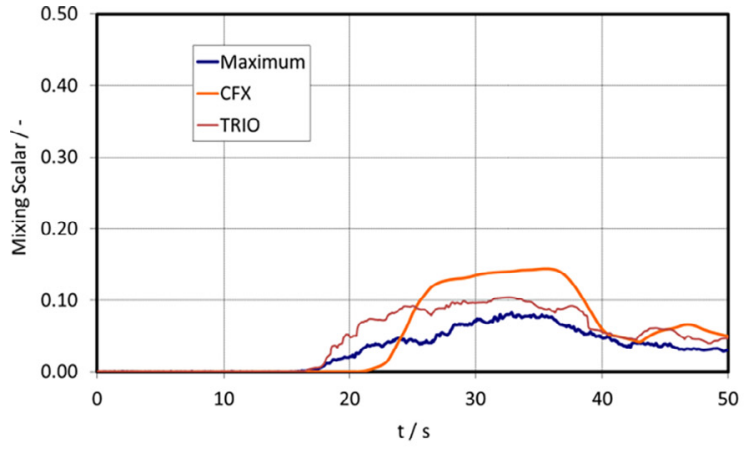

Maximum Mixing Scalar

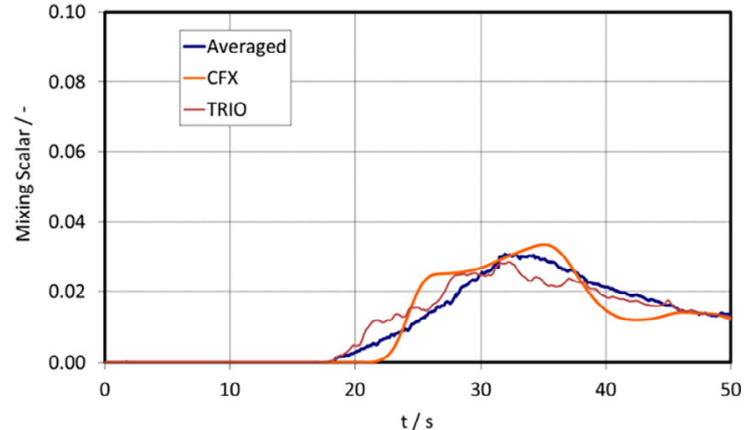

Averaged Mixing Scalar

Fig. 22. Time dependent tracer distributions at the lower downcomer sensor.

the cold leg $n^{\circ} 1$ and the downcomer has been stabilized by a preceding transient. Starting from a stagnant fluid, a constant inflow velocity was imposed at cold leg $n^{\circ} 1$ before the onset of the ECC injection: for about $10 \mathrm{~s}$ in CFX and for about $20 \mathrm{~s}$ in TrioCFD. For both codes, the time $\mathrm{t}=0 \mathrm{~s}$ is set to the instant $5 \mathrm{~s}$ before ECC injection onset. The initial scalar field was set to 0 . In CFX as an initial guess of the turbulent kinetic energy and the dissipation rate the turbulent intensity of $5 \%$ and the eddy viscosity ratio of 10 was used.

\section{Results of the forced convection experiment}

\subsection{Qualitative computational results}

The density difference is set to zero for the case d00m15 what results in $\mathrm{Fr}=\infty$. This Froude number characterizes pure momentum driven flow (see Fig. 5); there is no influence of the buoyancy force, neither to the global gravity forces nor to the turbulence buoyancy force. The flow-rate is set to $15 \%$ of the nominal mass flow rate of the cold leg, which was the highest flow rate used in the experimental studies. The first part of the transient calculation before ECC injection was used to establish a flow field in the cold leg and downcomer of ROCOM. During this period the ECC injection line was closed. The ensuing flow in cold leg $\mathrm{n}^{\circ} 1$ creates a momentum-controlled flow entering the downcomer. It is divided into two flow branches flowing in a downwards-directed helix around the core barrel (Fig. 11). A similar behavior was observed during nominal flow conditions using one pump (Cartland Glover et al., 2007).

The flow pattern in the cold leg is not changing significantly after the onset of ECC injection because there are no buoyancy forces present. The water from the ECC injection line first hits the core barrel at the opposite wall of cold leg $n^{\circ} 1$; this is caused by the momentum of the injected jet (Fig. 12). During the period of injection and after completion of the injection, the cold leg flow transports the ECC water towards the reactor inlet. As in the experiment, the area covered by the ECC water is first larger below the inlet nozzle. Since the momentum-driven flow is present all the time, the ECC water is then transported laterally all along the downcomer to the opposite side of cold leg $n^{\circ} 1$. This transport of ECC water to the opposite side of the injection is visible in Figs. 13 and 14. The mixing scalar $(0<\Theta<0.4)$ is shown for the instances $18 \mathrm{~s}$ and $20 \mathrm{~s}$. This phenomenon is more expressed in the LES 


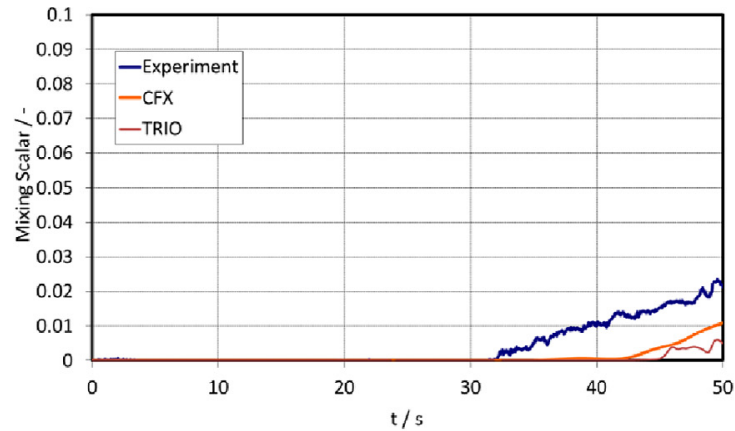

Local Mixing Scalar Pos. $22.5^{\circ}$

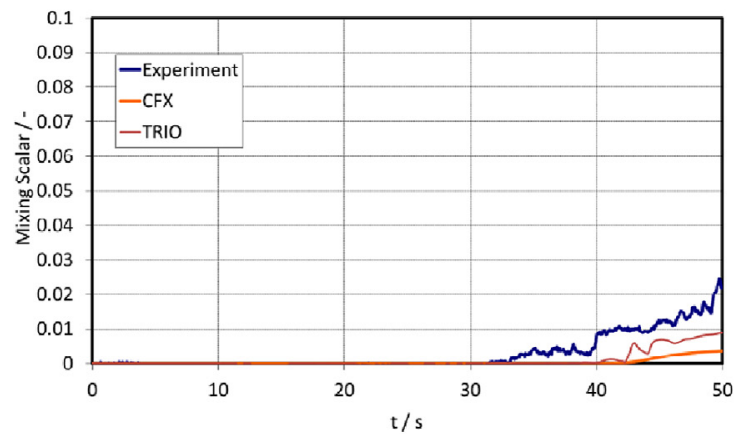

Local Mixing Scalar Pos. $11.25^{\circ}$
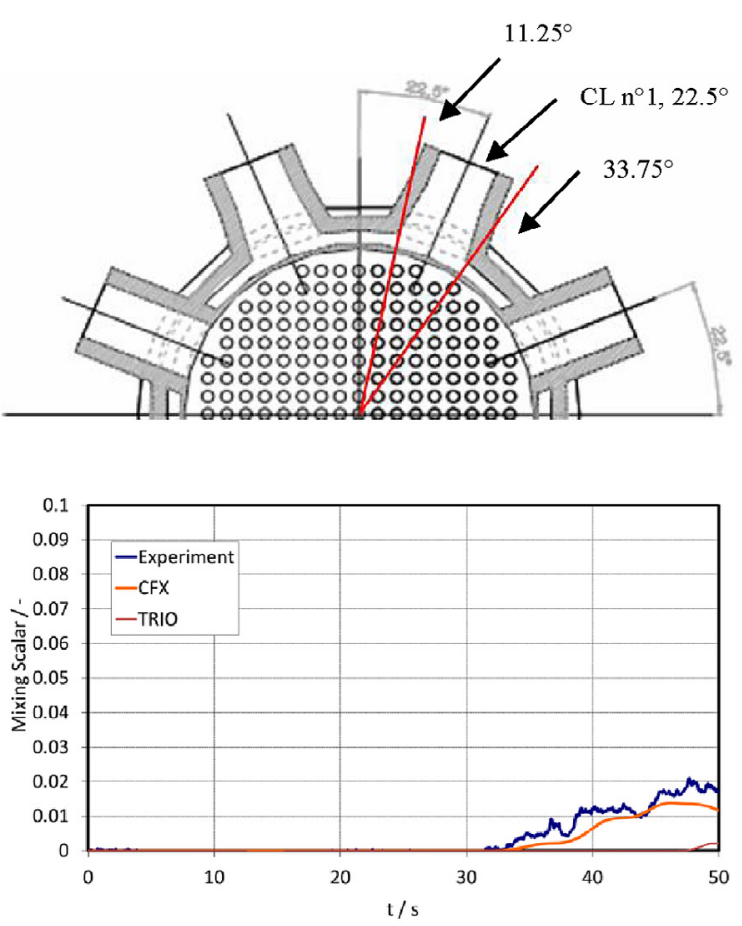

Local Mixing Scalar Pos. $33.75^{\circ}$

Fig. 23. Local transient tracer distributions at the lower downcomer sensor.

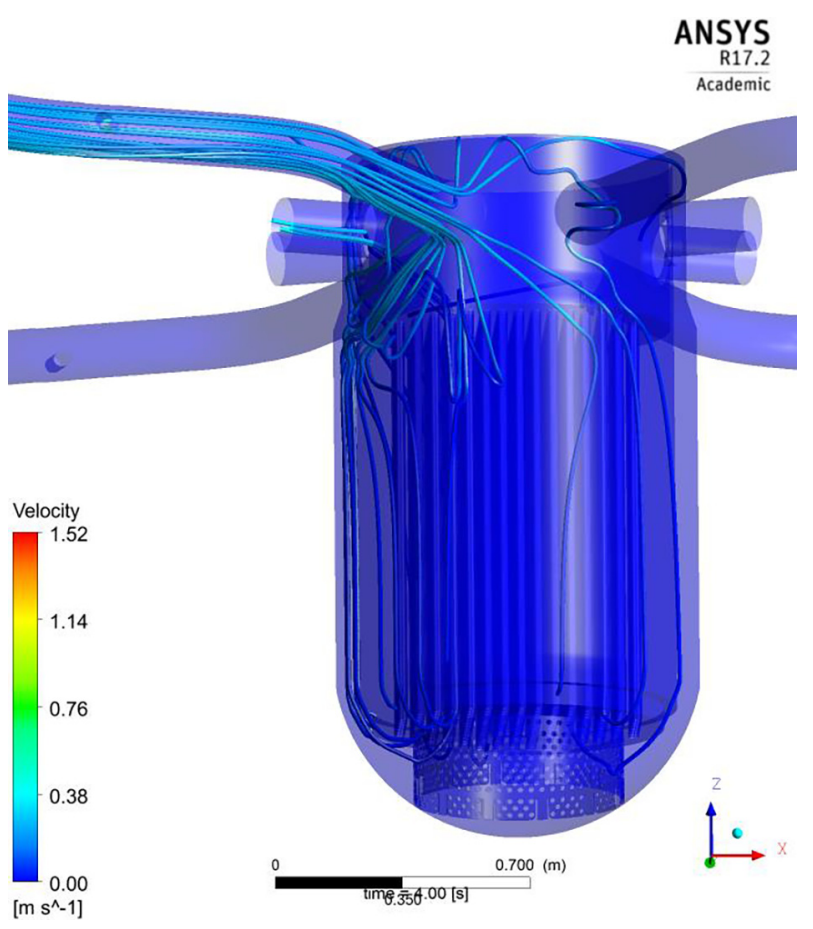

Fig. 24. Streamlines representing the fluid flow before the injection takes place (4 s), CFX-calculation.

calculation. This detected difference between the codes was not further analyzed. Possible reasons are the different time periods of flow initialization or the used less diffusive numerical schemes for LES.

Fig. 15 shows the temporal development of the mixing scalar in the upper and lower downcomer sensor (unwrapped presentation). The experiment and the CFX calculation are compared. The maximum experimental value of the mixing scalar in the upper downcomer is 0.12 (CFX 0.15, TrioCFD 0.13) and in the lower downcomer 0.083 (CFX

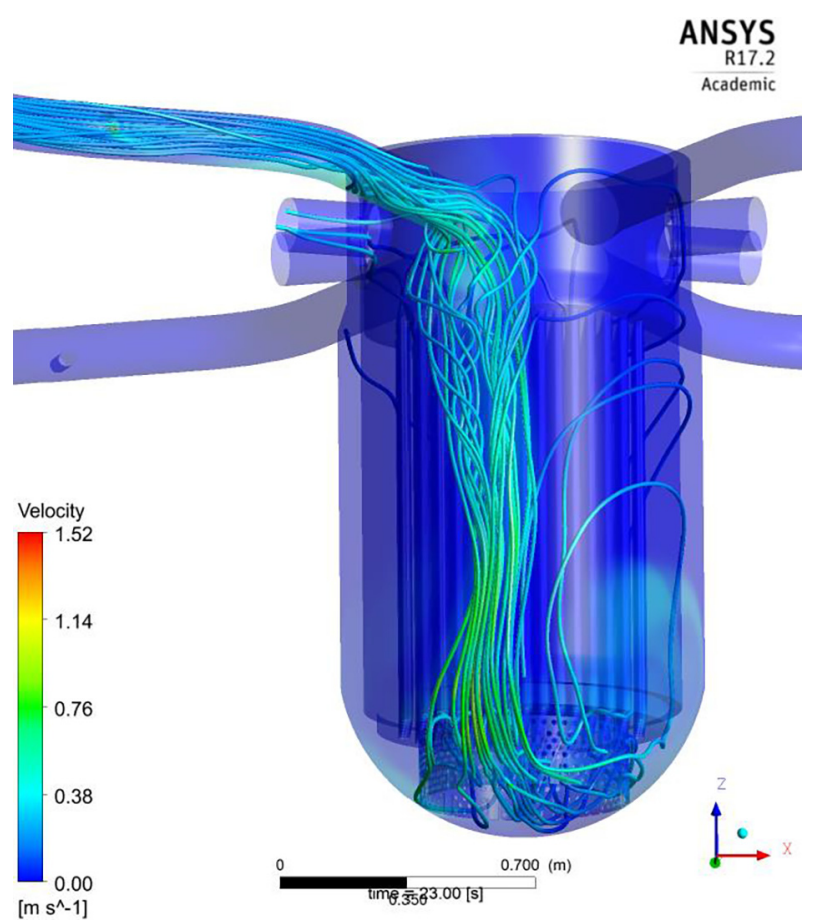

Fig. 25. Streamlines representing the fluid flow after the injection took place (23 s), CFX-calculation.

0.14, TrioCFD 0.10). In the CFX calculation the mixing and stratification in the cold leg causes the slug to be less diffuse, an effect, which results in the over prediction of the concentration at all positions of the wire mesh sensors compared to the experimental values. This is due to the fact that the slug of water in the simulation maintains a similar maximum value between the upper downcomer, whilst the concentration of the experimental slug decreases, as it is weakly dispersed. 

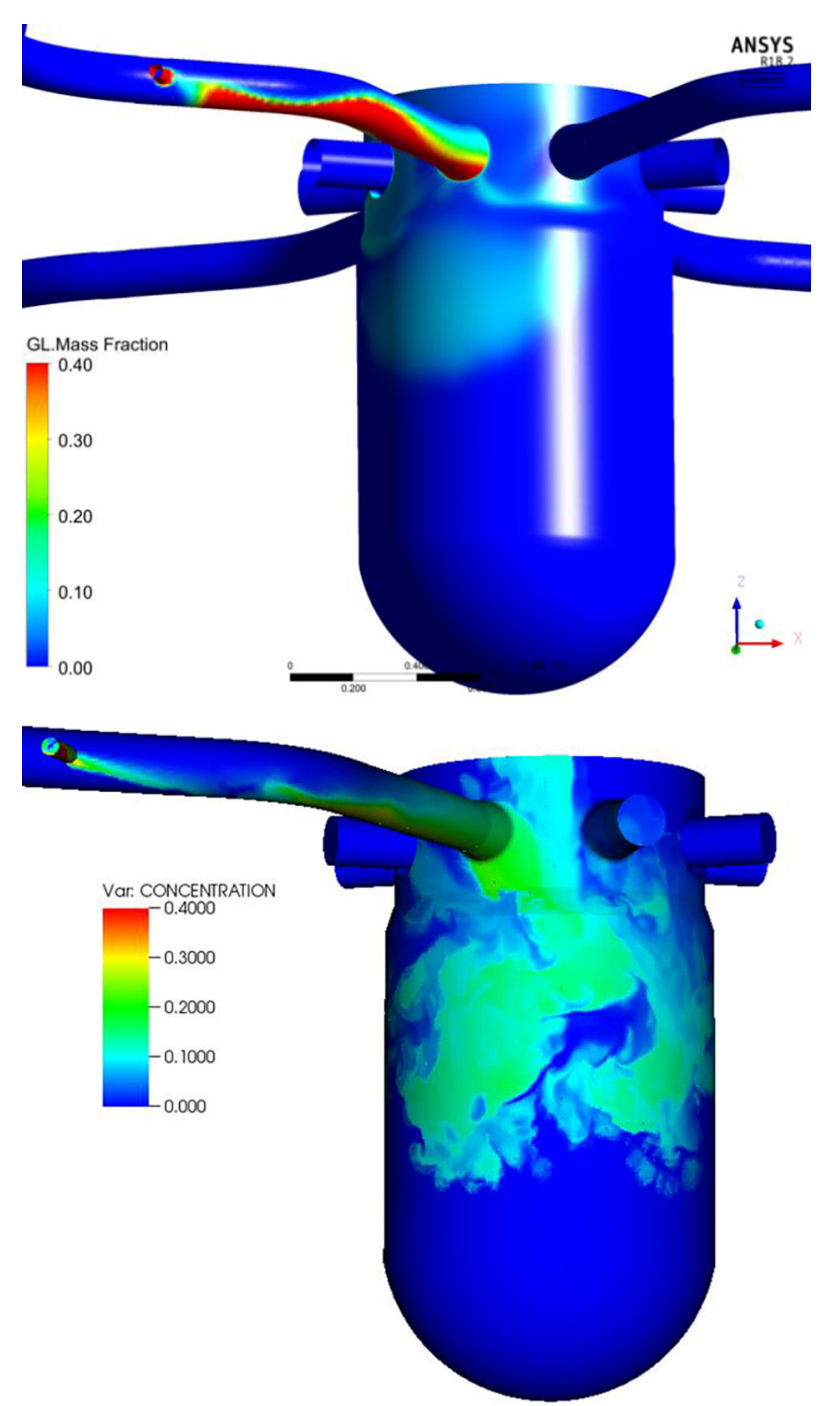

Fig. 26. Tracer distribution in the downcomer ( $18 \mathrm{~s}$ ), upper figure CFX, lower figure TrioCFD.

However, it is important to note that the timescales with which the slug is transported through the inlet nozzle and the upper downcomer are quite comparable and that there is a slight delay on the lower downcomer.

Fig. 16 shows the distribution of the ECC water in the cold leg $n^{\circ} 1$ at $\mathrm{t}=10 \mathrm{~s}$, i.e. $5 \mathrm{~s}$ after ECC injection onset. The cold water from the ECC injection line first hits the opposite wall of cold leg $n^{\circ} 1$; this is caused by the momentum of the injected jet. The transport of ECC water from the injection line to the downcomer and the subsequent beginning of azimuthal filling of the downcomer is well visible. During the downward flow, the ECC water in the downcomer is well mixed with the ambient coolant. The distribution of the ECC water in the lower part of the downcomer and at the core inlet is shown in Fig. 17 for $\mathrm{t}=34 \mathrm{~s}$. The location of the nozzle of cold leg $n^{\circ} 1$ is marked by the arrow. The CFX calculation shows a more homogeneous mixing of ECC water with the downcomer inventory than the TrioCFD calculation. The ECC water enter the core from the opposite side of cold leg $n^{\circ} 1$ in the LES calculation whereas the ECC water enters the core essentially at the side of cold leg $n^{\circ} 1$ in the CFX calculation. The first maximum of the ECC water arrives in the experiment at the opposite side of the injection loop, later ECC water appears also below the injection loop. Finally, the core inlet is homogeneously covered by the tracer.
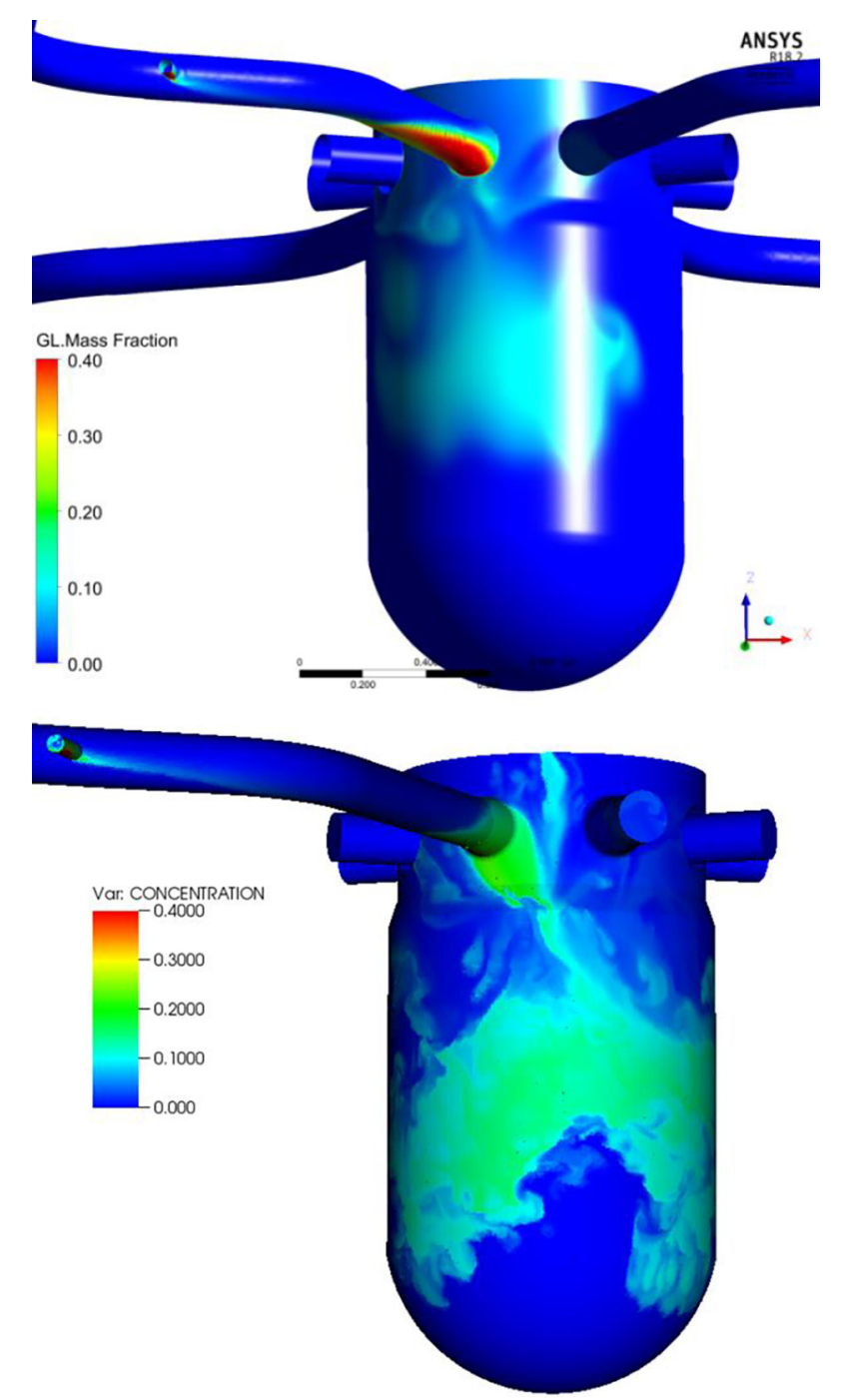

Fig. 27. Tracer distribution in the downcomer (20 s), upper figure CFX, lower figure TrioCFD.

\subsection{Quantitative computational results}

Turbulent, forced convection flows with small driving forces are characterized by hydraulically instable conditions what obviously yield in temporal unsteady flow fields. A local quantitative comparison of experiments and calculations is therefore difficult. The general agreement of the courses of the dominant quantities is the only way to asses a calculation. Local discrepancies in the transient courses are inevitably present in such comparisons due to statistical turbulent mixing effects.

\subsubsection{Analysis of the flow at the sensor in the cold leg $n^{\circ} 1$}

Fig. 18 shows a quantitative comparison of the ECC water concentrations in the cold leg sensor plane. Maximum and average mixing scalar (top figures) as well as two local measurement points of the cold leg sensor (bottom figures) are selected for the comparison. The local measurements are compared to the CFX calculation. The locations of the sensors are added to Fig. 18. Local values of the LES calculation are not available. The predicted local mixing scalar agrees well with the experimental values, especially the moment of arriving of the ECC water at the sensor plane is well predicted.

For both codes, the temporal course of the maximum of the mixing scalar is compared to the measured one for the location of the cold leg sensor. This comparison is shown on top of Fig. 18. Additionally, for 


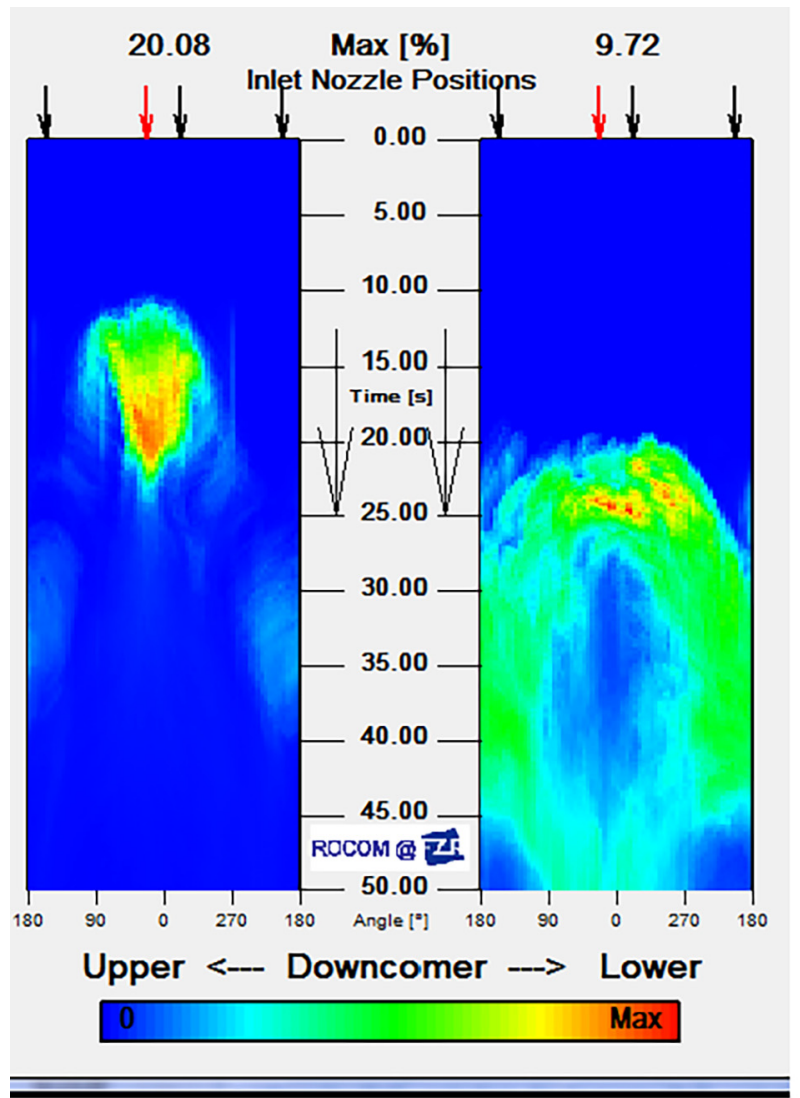

Experiment

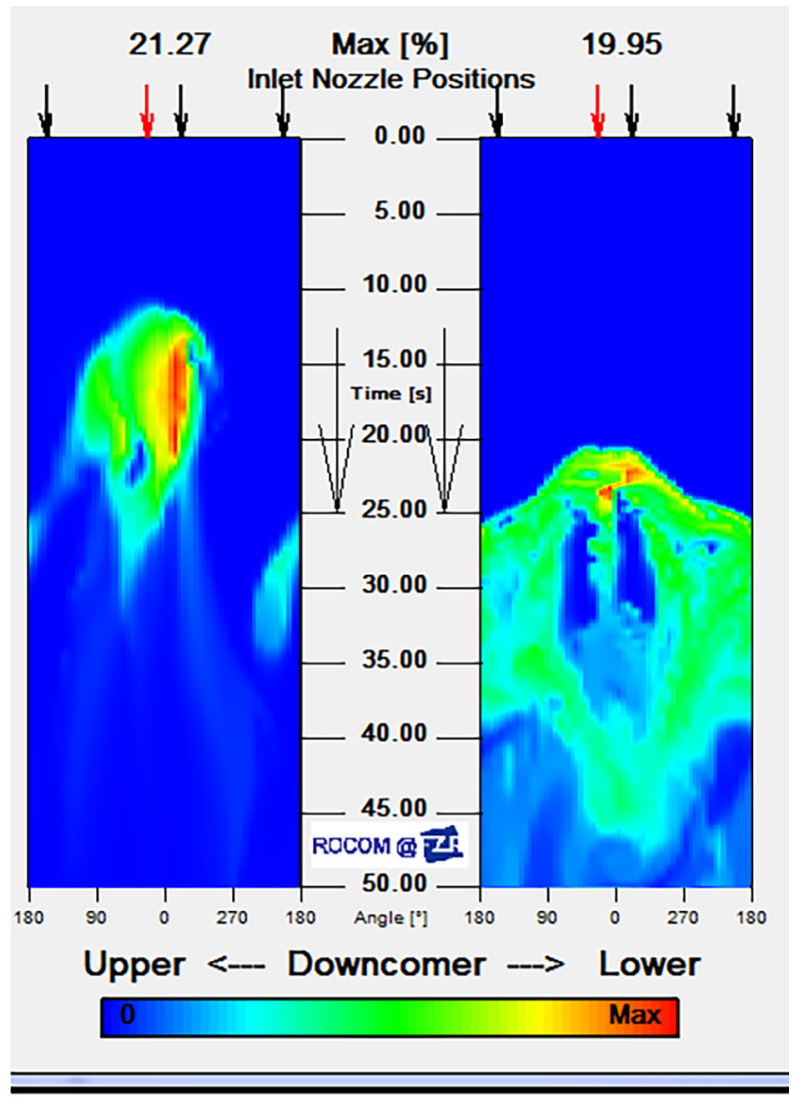

CFX

Fig. 28. Time dependent tracer distributions at the Upper and Lower Downcomer Sensor, left hand side Experiment, right hand side CFX.

both codes and the measurement, the average mixing scalar is calculated according to Eq. (3) at each time step for the cold leg sensor plane:

$\bar{\theta}=\frac{1}{N_{p}} \sum_{i=1}^{N_{p}} \theta_{i}$

$\mathrm{N}_{\mathrm{p}}$ is the number of calculation points in the mesh sensor plane, measuring points respectively. The average values are also compared in Fig. 18. The temporal courses of calculated maximum and average value are in good accordance to the experiment with a negligible overestimation of the maximum concentration by CFX.

\subsubsection{Analysis of the flow at the upper downcomer sensor}

The instantaneous azimuthal distribution of the mixing scalar is shown in Fig. 19. Here, 32 circumferential sensor positions in the middle of the upper and lower downcomer sensor plane were selected for the comparison of data. The distribution is given for the instances $13 \mathrm{~s}$ and $17 \mathrm{~s}$ for the upper downcomer sensor and for the instances $22 \mathrm{~s}$ and $26 \mathrm{~s}$ for the lower downcomer sensor. For a better understanding, the azimuthal direction of the sensor positions is added to the top of the figure. The arrangement of the cold leg nozzles $n^{\circ} 1$ and $n^{\circ} 4$ is also added to the figure. In general, both codes represent correctly the instantaneous azimuthal distribution of the mixing scalar in the downcomer. Discrepancies can not only be addressed to code and modelling differences but also to the unsteady and stochastic character of turbulent flow.

Fig. 20 shows the temporal variation of the maximum and averaged value of the mixing scalar, calculated for the 32 positions in the middle of the upper downcomer sensor plane. The average value is calculated according to Eq. (3). The agreement to the experiment is good for both turbulence modelling approaches (RSM and LES). In particular, the moment is kept well when the slug reaches the first time the measuring plane. Both codes capture well the temporal evolution of the average value. Difference between codes and experiment are related to variations in the radial distribution of the mixing scalar as well as to azimuthal variations. Minor deviations occur at the maximum value.

The dynamic of the descending slug can also be seen in the time course of the local mixing scalar at the upper downcomer sensor (Fig. 21). Three azimuthal positions in the region below the inlet nozzle $\mathrm{n}^{\circ} 1$ were selected, which are located radially in the center of each downcomer sensor plane. The selected circumferential angles of $22.5^{\circ}$ (center of the cold leg ${ }^{\circ} 1$ ), $11.25^{\circ}$ and $33.75^{\circ}$ are also shown in Fig. 21.

The LES underestimates the mixing scalar at locations below loop $\mathrm{n}^{\circ} 1$ due to the strong effect of forced convection shown in Figs. 13 and 14.

\subsubsection{Analysis of the flow at the lower downcomer sensor}

The maximum and average values of the mixing scalar at the lower downcomer sensor, which are presented in Fig. 22 on a zoomed mixing scalar scale, are calculated for the 32 positions in the middle of the lower downcomer sensor plane (analogue to those of the upper downcomer sensor). At the lower downcomer sensor, the maximum values of the mixing scalar are significantly smaller than in the upper downcomer (Fig. 20) due to turbulent mixing in the downcomer. As a consequence, this mixing leads to a larger area, which is covered by the slug. Both codes get well the average value. Discrepancies in the maximum value should not be overestimated due to the unsteady character of turbulent mixing.

This behavior of the codes is confirmed by the local mixing scalar concentration in the middle of the downcomer at the $22.5^{\circ}, 11.25^{\circ}$ and $33.75^{\circ}$ circumferential positions (Fig. 23).

Both codes have difficulties in reproducing correctly these local 


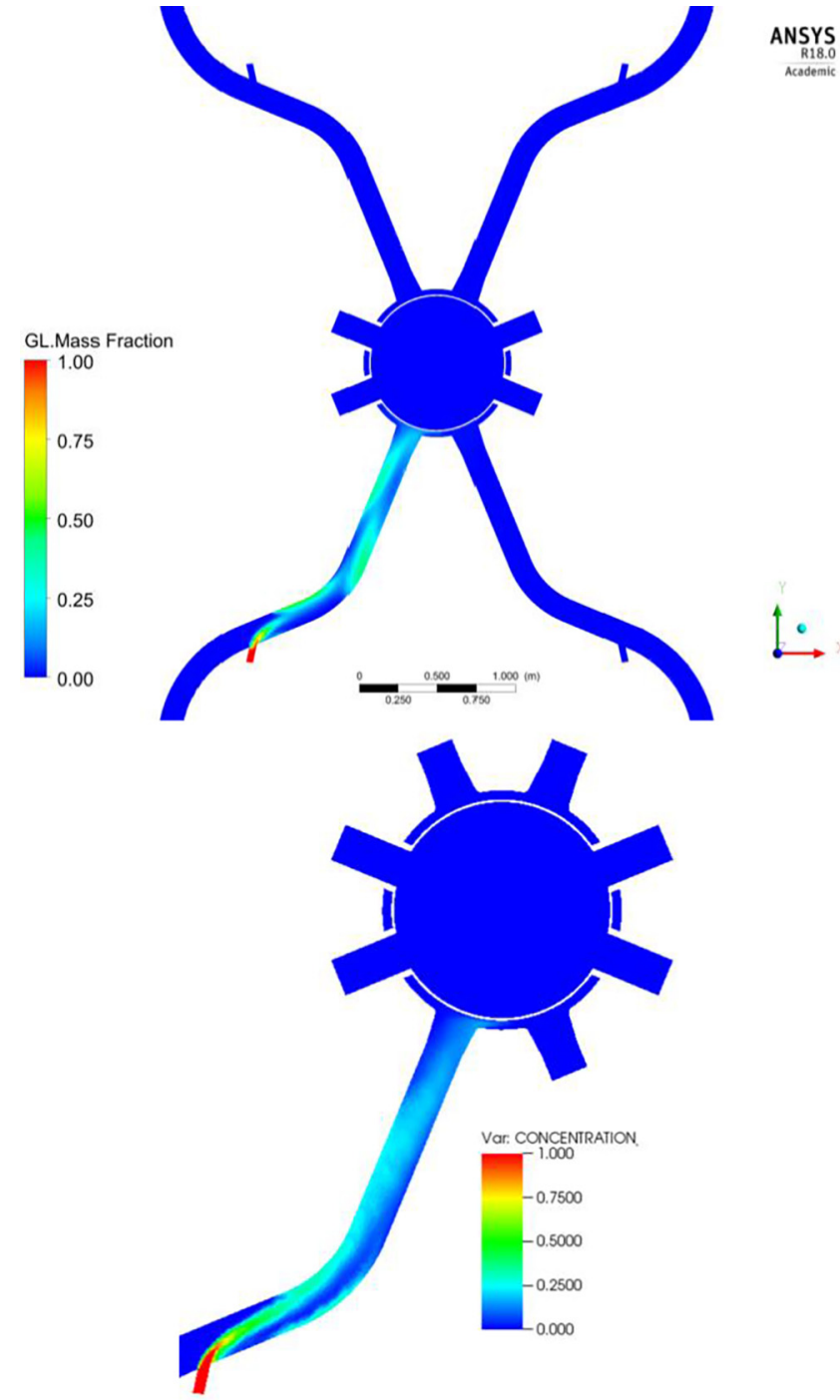

Fig. 29. Tracer distribution in the cold leg (10 s), upper figure CFX, lower figure TrioCFD.

values. As can be seen from Fig. 15, only a small part of the tracer arrives below cold leg $n^{\circ} 1$, representing very low mixing scalar concentrations around 0.02 (value taken from Fig. 23). The majority of the tracer is transported downward to the lower plenum at the opposite side of leg $n^{\circ} 1$. The tracer arrives at the lower downcomer below leg $n^{\circ}$ 1 only after having crossed the lower plenum. Thus, the incapacity of the codes to predict in detail this complex tracer track is not surprising.

\section{Results of the mixed convection experiment}

\subsection{Qualitative computational results}

As already mentioned, the density difference between ECC injection and RPV inventory is set to $10 \%$, what results in $\mathrm{Fr}=0.8208$. This Froude number characterizes mixed convection (see Fig. 5) and represents the transition region between momentum driven and density driven flow. The flow rate is set to $10 \%$ of the nominal mass flow rate in the cold leg. The first part of the transient calculation before ECC injection was used to establish a flow field in the cold leg and downcomer of ROCOM. During this period the ECC injection line was closed. The ensuing flow in cold leg $\mathrm{n}^{\circ} 1$ creates first a momentum-controlled flow entering the downcomer as in the case d00m15 (Fig. 11). The main flow is divided into two branches flowing in a downwards-directed helix

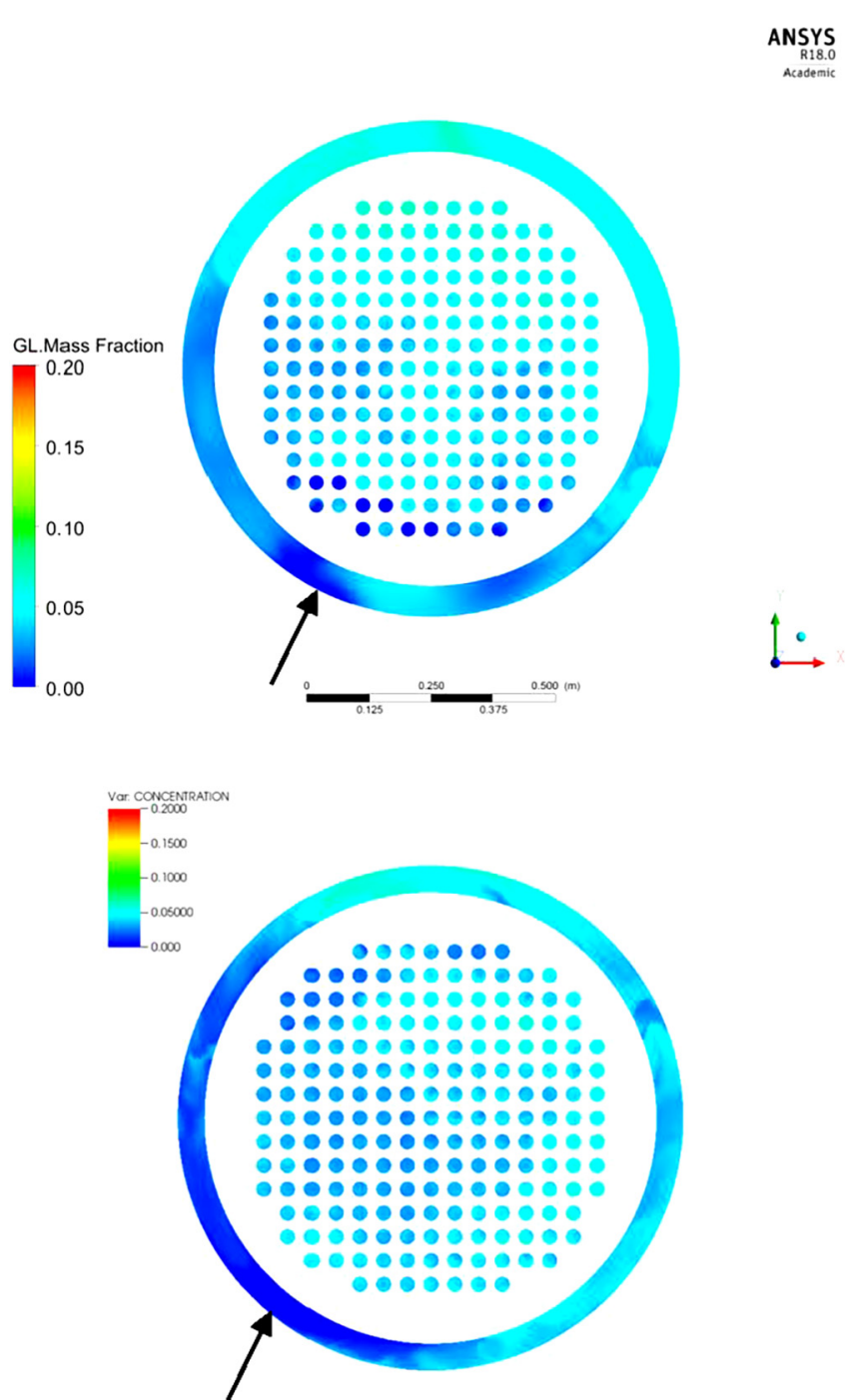

Fig. 30. Tracer distribution in the core inlet ( $34 \mathrm{~s}$ ), upper figure CFX, lower figure TrioCFD.

around the core barrel as shown in Fig. 24.

After the onset of ECC injection with cold (i.e. saccharine) water, the flow pattern in the cold leg changes because of buoyancy forces. The cold water from the ECC injection line first hits the core barrel at the opposite wall of cold leg $n^{\circ} 1$; this is caused by the momentum of the injected jet (Fig. 25). Later, the ECC water is partly mixing with the ambient loop inventory, but mainly propagating downward towards the downcomer bottom forming a wide streak below the cold leg nozzle.

The mixing scalar in the ROCOM RPV is shown in Figs. 26 and 27 for the instances $18 \mathrm{~s}$ and $20 \mathrm{~s}(0<\Theta<0.4)$. At the beginning of the transient, the area covered by the ECC water is bigger below the inlet nozzle because the momentum-driven flow field is still present (Fig. 26). However, because of the density differences between the ECC water and the ambient coolant, the initial momentum-controlled flow in the downcomer starts changing. At later times a density-dominated flow is established (Fig. 27). The ECC water creates a downward streak in the downcomer. The ECC water distribution in the downcomer calculated by RSM and LES are quite different. The ECC plume spreads azimuthally more in LES and is more deformed by turbulence than in the RSM calculation.

In the calculations, this streak is not stable. It starts fluctuating at half of the downcomer height in the CFX calculation. During this downward flow, the ECC water in the downcomer is well mixed with 


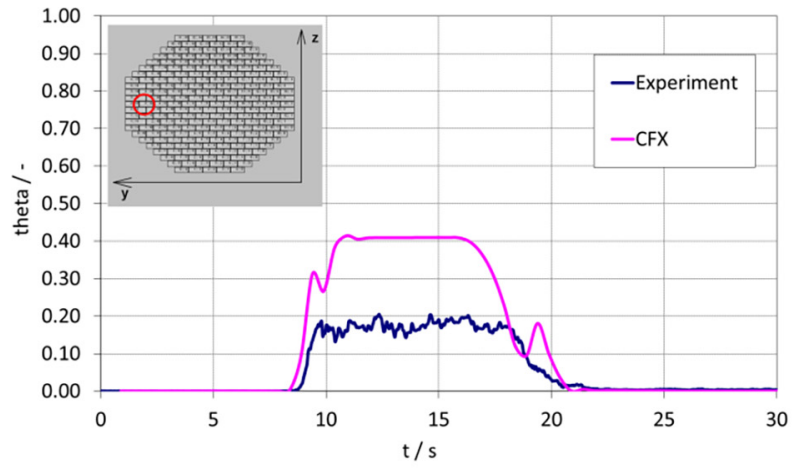

Local Mixing Scalar Pos. 0312

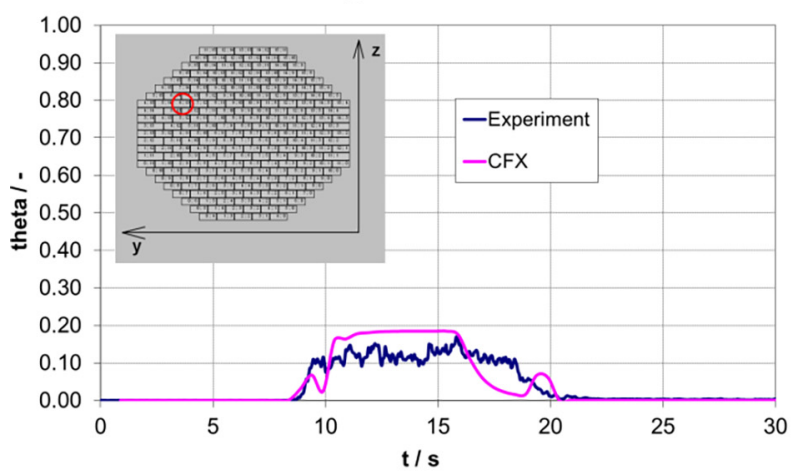

Local Mixing Scalar Pos. 0613

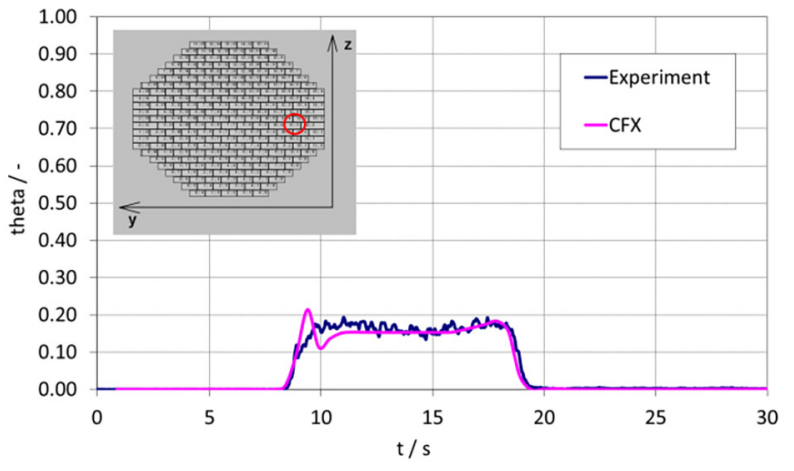

Local Mixing Scalar Pos. 1103

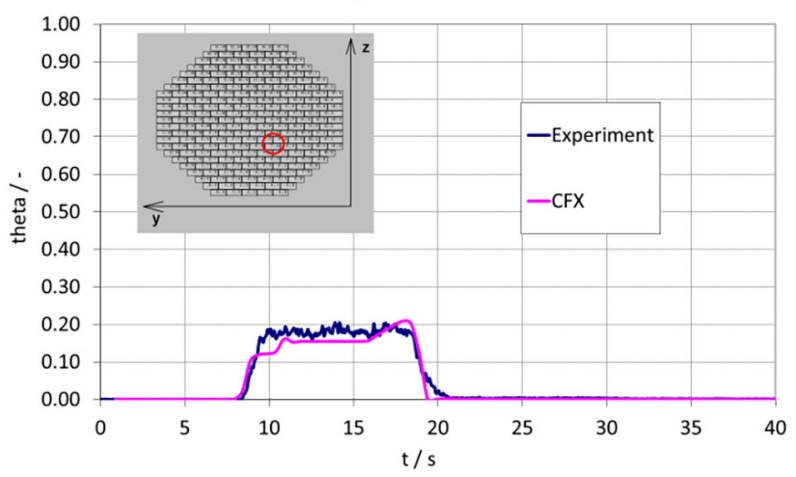

Local Mixing Scalar Pos. 0704

Fig. 31. Time dependent tracer course at the cold leg inlet nozzle sensor.

the ambient coolant. When the plug reaches the lower plenum, it swaps to the opposite side of the injection loop. The lower plenum is filled with already well-mixed ECC water.

Fig. 28 shows the temporal development of the mixing scalar in the upper and lower downcomer sensor (unwrapped presentation). The experiment (left) and the CFX calculation (right) are compared. The maximum experimental value of the mixing scalar in the upper downcomer is 0.20 (CFX 0.21, TrioCFD 0.21) and in the lower downcomer 0.097 (CFX 0.20, TrioCFD 0.13). In the CFX calculation the mixing and stratification in the cold leg causes the slug to be less diffuse, an effect, which results, compared to the experimental values, in the over prediction of the concentration at all positions of the wire mesh sensors. This is due to the fact that the slug of water in the simulation maintains a similar maximum value between the upper downcomer, whilst the concentration of the experimental slug decreases, as it is weakly dispersed. However, it is important to note that the timescales with which the slug is transported through the inlet nozzle and the upper downcomer are quite comparable and that there is only a slight delay on the lower downcomer.

Fig. 29 shows the distribution of the ECC water in the cold leg ${ }^{\circ} 1$ at $\mathrm{t}=10 \mathrm{~s}$. The transport of ECC water from the injection line to the downcomer is visible. The cold water from the ECC injection line first hits the opposite wall of cold leg $n^{\circ} 1$; this is caused by the momentum of the injected jet. The slug enters the downcomer with a slight angular moment in counter clockwise direction. During the downward flow, the ECC water in the downcomer is mixed with the ambient coolant. The distribution of the ECC water in the lower part of the downcomer and at the core inlet is shown in Fig. 30 for $t=34 \mathrm{~s}$. The location of the nozzle of cold leg $n^{\circ} 1$ is marked by the arrow. Both codes show a homogeneous mixing of ECC water with the RPV inventory. The ECC water enter the core starting from the opposite side of cold leg $n^{\circ} 1$ but rapidly covers the whole core inlet.

\subsection{Quantitative computational results}

\subsubsection{Analysis of the flow at the sensor in the cold leg $n^{\circ} 1$}

A quantitative comparison of measured and calculated mixing scalar concentration in the cold leg sensor plane is performed. The mixing scalar is injected with the ECC water. Fig. 31 shows the transient concentration course at four measurement points at the cold leg sensor for the comparison of the experiment to the CFX calculation. The locations of the local sensor points are added to Fig. 31. Local data of the LES calculations are not available at the inlet nozzle sensor. The predicted mixing scalar agrees well with the experimental values at three out of four positions as shown in Fig. 31. Especially the moment of arriving of the ECC water at the sensor plane is well represented. The calculated mixing scalar is overestimated only at the local position 0312 .

\subsubsection{Analysis of the flow at the upper downcomer sensor}

The instantaneous azimuthal distribution of the mixing scalar is shown in Fig. 32. Here, 32 circumferential sensor positions in the middle of the upper and lower downcomer sensor plane were selected for the comparison of data. The distribution is given for the instances $13 \mathrm{~s}$ and $21 \mathrm{~s}$ for the upper downcomer sensor and for the instances $22 \mathrm{~s}$ and $30 \mathrm{~s}$ for the lower downcomer sensor. For a better understanding, the azimuthal direction of the sensor positions as well as their locations along a circle is added to the top of the Fig. 32. The arrangement of the cold leg nozzles $n^{\circ} 1$ and $n^{\circ} 4$ is also added to the figure. In general, both codes represent correctly the instantaneous azimuthal distribution of the mixing scalar in the downcomer. Due to buoyancy forces, the heavier ECC water is mainly located at the upper downcomer sensor below the nozzles of the cold legs $n^{\circ} 1$ and $n^{\circ} 2$. At the lower downcomer sensor, the whole downcomer is filled successively by ECC water. As in the forced convection case (d00m15), discrepancies can not only be addressed to code and modelling differences but also to the unsteady and stochastic character of turbulent flow. 

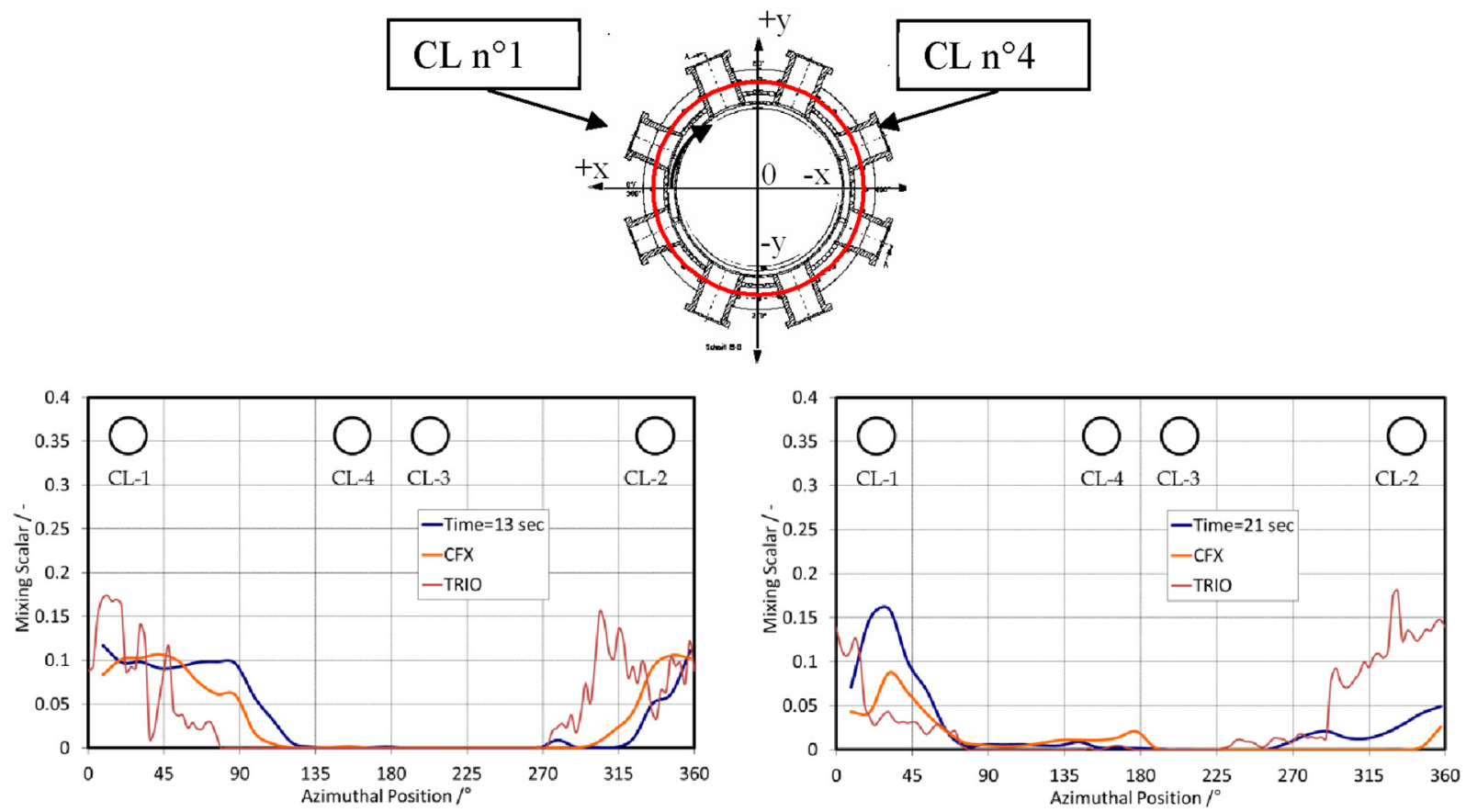

Upper downcomer Sensor
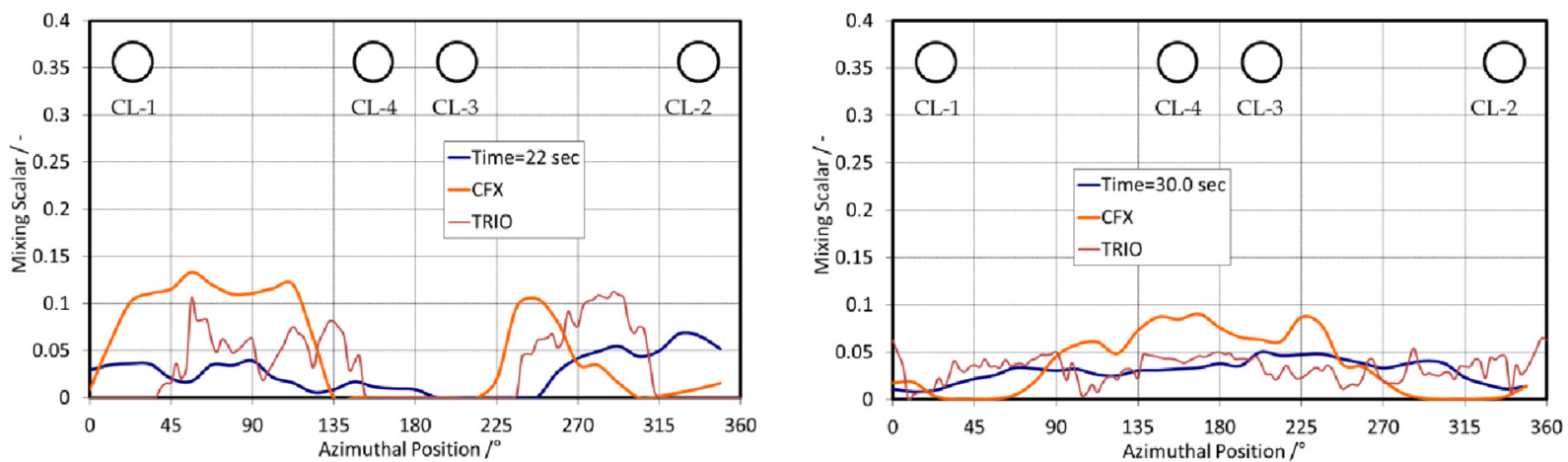

Lower downcomer Sensor

Fig. 32. Instantaneous circumferential tracer distributions for the 32 positions in the center of the upper and lower downcomer sensor.

Fig. 33 shows the temporal variation of the maximum and averaged value of the mixing scalar, calculated for the 32 positions in the middle of the upper downcomer sensor plane. The agreement to the experiment is good for both turbulence modelling approaches (RSM and LES). In particular, the moment is kept well, when the slug reaches the first time the measuring plane. Both codes capture the mean value. Deviations

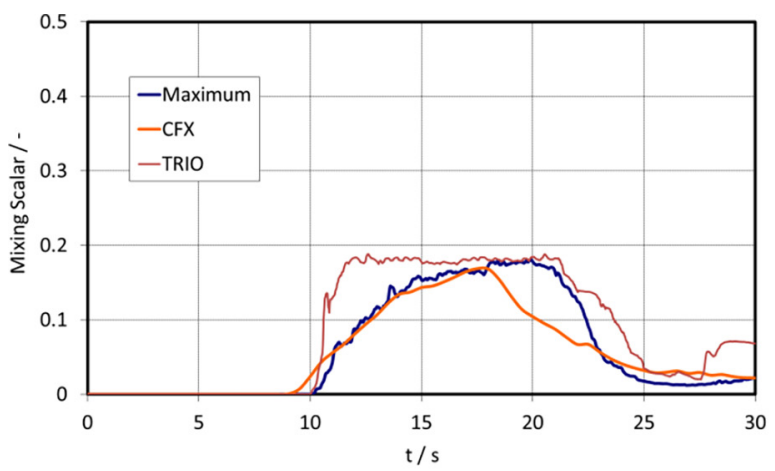

Maximum Mixing Scalar occur at the maximum value. As already stated for the $\mathrm{d} 00 \mathrm{~m} 15$ case, this discrepancy should not be overestimated.

The dynamic of the descending slug can also be seen in the time course of the local mixing scalar at the upper downcomer sensor (Fig. 34). Three azimuthal positions in the region below the inlet nozzle $\mathrm{n}^{\circ} 1$ were selected, which are located radially in the center of each

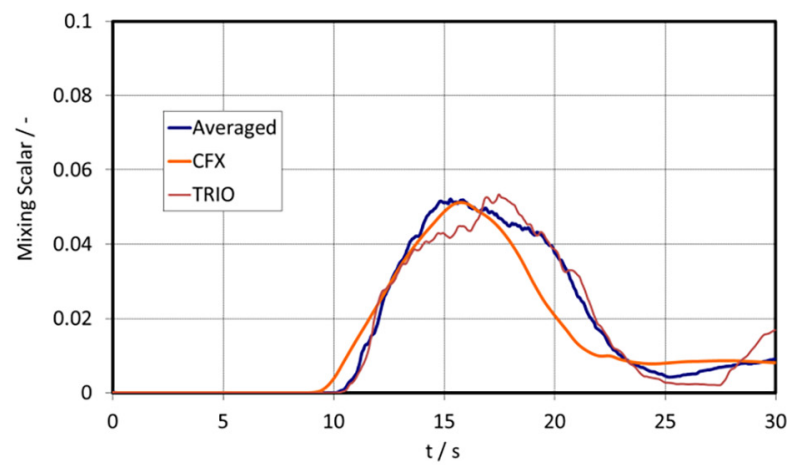

Averaged Mixing Scalar

Fig. 33. Transient tracer course at the upper downcomer sensor. 


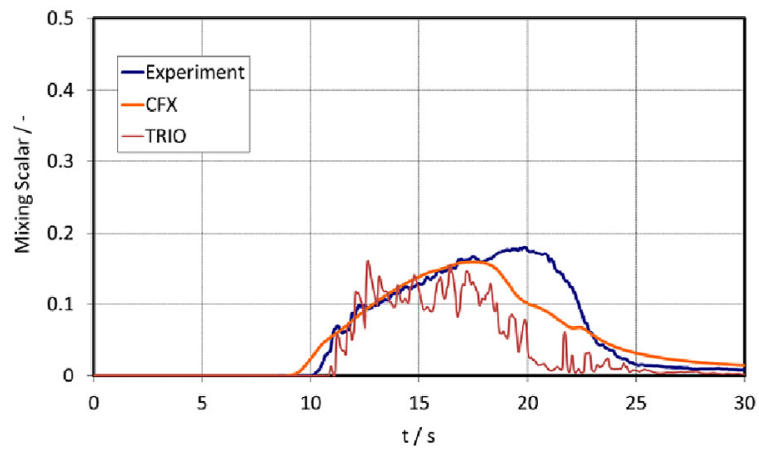

Local Mixing Scalar Pos. 22.5

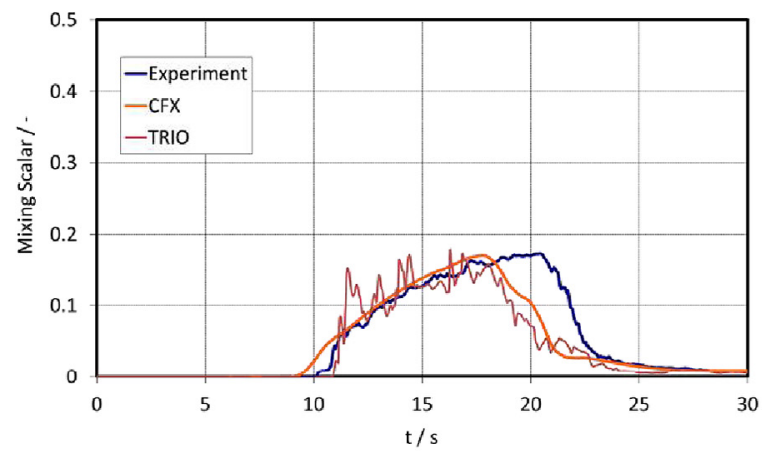

Local Mixing Scalar Pos. $11.25^{\circ}$
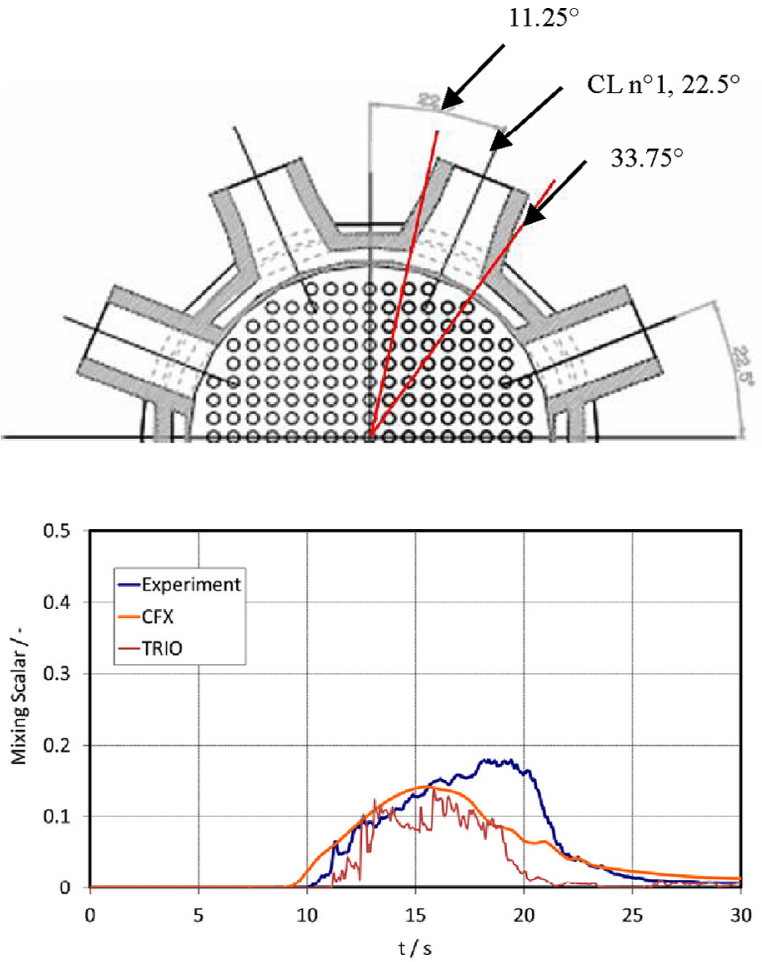

Local Mixing Scalar Pos. $33.75^{\circ}$

Fig. 34. Local transient tracer distributions at the upper downcomer sensor.

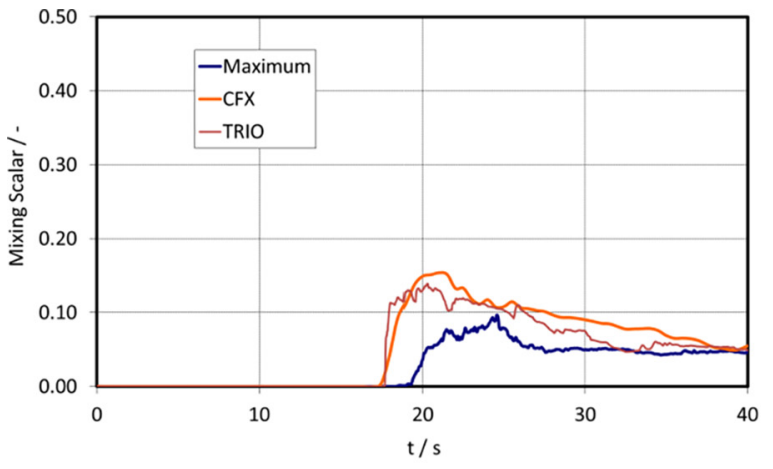

Maximum Mixing Scalar

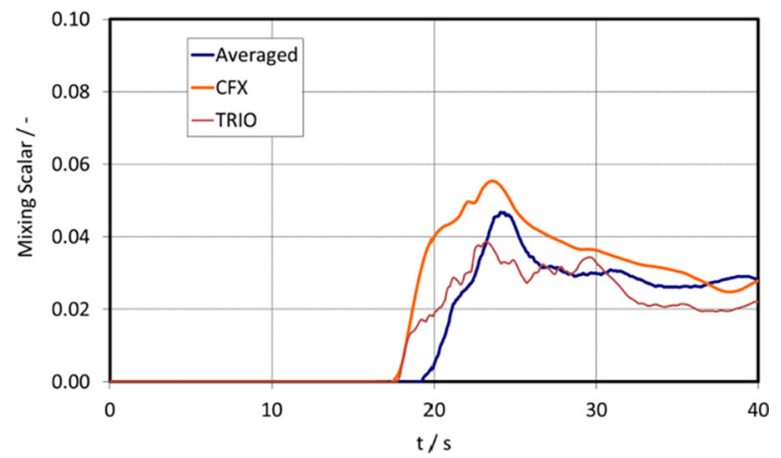

Averaged Mixing Scalar

Fig. 35. Time dependent tracer course at the lower downcomer sensor.

downcomer sensor plane. The selected circumferential angles of $22.5^{\circ}$ (center of the cold $\operatorname{leg} n^{\circ} 1$ ), $11.25^{\circ}$ and $33.75^{\circ}$ are also shown in Fig. 34.

\subsubsection{Analysis of the flow at the lower downcomer sensor}

The maximum and average values of the mixing scalar at the lower downcomer sensor, which are presented in Fig. 35, are calculated for the 32 positions in the middle of the lower downcomer sensor plane (analogue to those of the upper downcomer sensor). At the lower downcomer sensor, the maximum values of the mixing scalar are significantly smaller than in the upper downcomer (Fig. 33) due to turbulent mixing in the downcomer. As a consequence, this mixing leads to a larger area, which is covered by the slug. The moment when the slug arrives at the sensor plane is predicted about $2 \mathrm{~s}$ too early in both calculations, probably due to an overestimation of the plume downward velocity. Both codes get rather well the average value (please notice the zoomed mixing scalar scale in Fig. 35) but over-predict the maximum value.

This behavior of the codes is confirmed by the local mixing scalar concentration in the middle of the downcomer at the $22.5^{\circ}, 11.25^{\circ}$ and $33.75^{\circ}$ circumferential positions (Fig. 36). Both codes get approximately correct the temporal course of the experimentally determined concentration. However, differences exist in the dynamic of the passage of the slug.

\section{Summary and conclusion}

This paper shows results of post-test numerical simulations of ROCOM PTS experiments, one of them proposed by IAEA as an international open benchmark. Experimental data obtained for a constant flow rate in one loop, which represents the magnitude of natural circulation and $0 \%(\mathrm{~d} 00 \mathrm{~m} 15)$ respectively $10 \%(\mathrm{~d} 10 \mathrm{~m} 10)$ density difference between ECC and loop water inventory were compared to numerical predictions from the CFD software packages CFX and TrioCFD. The first comparison of these codes on ROCOM mixing experiments have been made in 2005 for pure buoyancy driven flow (d10m05).

Compared to the earlier study, significant progress was made in the 


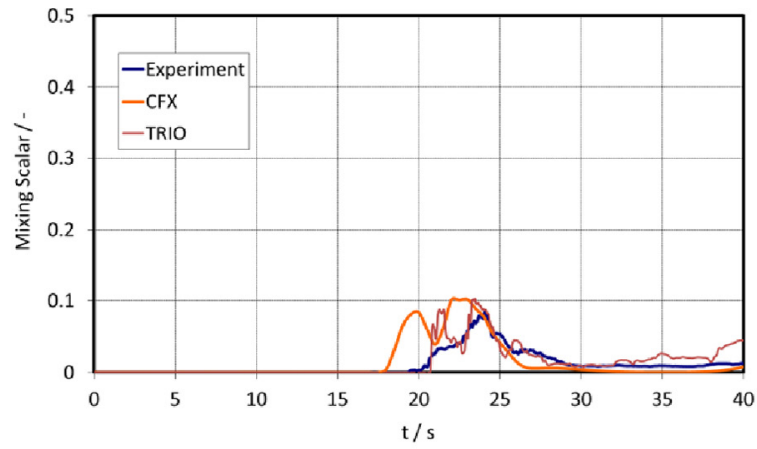

Local Mixing Scalar Pos. 22.5

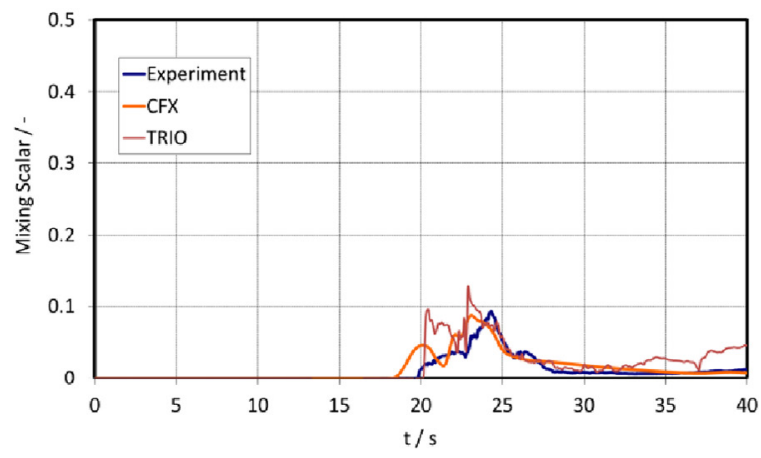

Local Mixing Scalar Pos. $11.25^{\circ}$
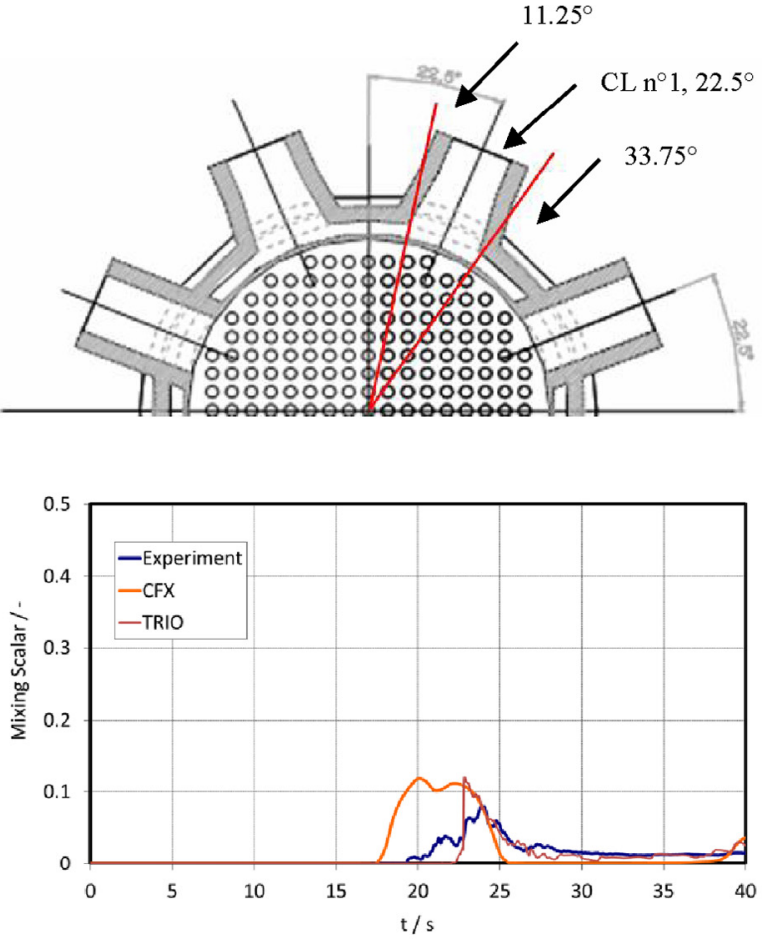

Local Mixing Scalar Pos. $33.75^{\circ}$

Fig. 36. Local transient tracer distributions at the lower downcomer sensor.

development of CFD codes concerning both numerical aspects and physical modelling; here especially the treatment of turbulence. Thus, the more challenging cases of mixed convection and momentum driven flow are analyzed in this paper by using the RSM of CFX and LES with TrioCFD.

Concerning the prediction of a PTS event (d10m10), both codes show good predictions of the ECC injection phase with the mixing of ECC water with the cold leg inventory. The rather good reproduction of the mixing scalar transient at the upper downcomer sensor shows the capacity of CFD to analysis single phase PTS events, even for the intermedia range between momentum- and density driven flow.

It has been observed experimentally in the ROCOM test facility and calculated by CFD that for the Froude number of $\mathrm{Fr}=0.85$ cold ECC water mixes only partly with the ambient inventory in the cold leg. A stratified flow is developing during the injection. In the downcomer, a momentum-driven flow field is present during the injection phase. At later times, when the injection is stopped, the flow becomes density dominated and the ECC water propagates vertically downwards in the downcomer. The differences between experiment and calculations increase with distance to the ECC injection, i.e. at the location of the lower downcomer. The calculated ECC slug arrives at this location with a short time lag. The azimuthal distribution of the ECC water at this location is very sensitive to the initial density ratio between ECC water and cold leg inventory.

For the momentum driven flow the Froude number is $\mathrm{Fr}=\infty$; there is no influence of buoyancy forces. The flow entering the downcomer is divided into two flow branches flowing in a downwards-directed helix around the core barrel. This flow field is almost identical before, during and after ECC injection. The momentum driven regime has caused problems for CFD to correctly predict the mixing scalar in the downcomer, what has not been expected previously.

Nevertheless, the following general conclusions can be drawn: Both codes (and turbulence modelling approaches) show a good qualitative agreement with the experimental data. The dominant mixing phenomena have been treated correctly. However, it seems that buoyancy forces must be calculated more precisely in the mixed convection regime. Further, experimental and numerical analysis seem necessary to better understand the flow behavior under momentum driven flow conditions at low velocities.

\section{Acknowledgments}

The TrioCFD analysis was granted access to the HPC resources of CINES under the allocation A0012A07571 made by GENCI.

\section{References}

Angeli, P.-E., Bieder, U., Fauchet, G., 2015. Overview of the Trio U code: main features, V $\& \mathrm{~V}$ procedures and typical applications to engineering. 16th International Topical Meeting on Nuclear Reactor Thermal Hydraulics, NURETH-16, Chicago, USA.

Angeli, P.-E., Puscas, M.-A., Fauchet, G., Cartalade, A., 2017. FVCA8 Benchmark for the Stokes and Navier-Stokes Equations with the TrioCFD Code-Benchmark Session. In: Finite Volumes for Complex Applications VIII - Methods and Theoretical Aspects, pp. $181-202$.

Ansys CFX, 2017. CFX User Manual. Ansys Inc.

Ansys ICEMCFD, 2017. ICEMCFD User Manual. Ansys Inc

Cartland Glover, G.M., Höhne, T., Kliem, S., Rohde, U., Weiss, F.-P., Prasser, H.-M., 2007. Hydrodynamic phenomena in the downcomer during flow rate transients in the primary circuit of a PWR. Nucl. Eng. Des. 237, 732-748.

Crouzieux, M., Raviart, P.A., 1973. Conforming and non-conforming finite element methods for the stationary Stokes equations. R.A.I.R.O Anal Numer. 7, 33-76.

Ducros, F., Bieder, U., Cioni, O., Fortin, T., Fournier, B., Fauchet, G., Quéméré, P., 2010 Verification and validation considerations regarding the qualification of numerical schemes for LES for dilution problems. Nucl. Eng. Des. 240 (9), 2123-2130.

Hirt, C.V., Nichols, B.D., Romero, N.C., 1975. SOLA - A Numerical Solution Algorithm for Transient Flow. Los Alamos National Lab. Report LA-5852.

Höhne, T., Kliem, S., Bieder, U., 2006. Modeling of a buoyancy-driven flow experiment at the ROCOM test facility using the CFD-codes CFX-5 and TRIO_U. Nucl. Eng. Design 236, 1309-1325.

Höhne, T., Kliem, S., Vaibar, R., 2009. Experimental and numerical modeling of transition matrix from momentum to buoyancy-driven flow in a pressurized water reactor. J. Eng. Gas Turbines Power - Trans. ASME 131, 012906.

Höhne, T., Kliem, S., 2016. IAEA CRP benchmark of ROCOM BORON DILUTION and PTS test cases for the use of CFD in reactor design, Computational Fluid Dynamics for Nuclear Reactor Safety Applications - CFD4NRS-6, Cambridge, USA.

Kliem, S., Suehnel, T., Rohde, U., Höhne, T., Prasser, H.-M., Weiss, F.-P., 2008. 
Experiments at the mixing test facility ROCOM for benchmarking of CFD-codes. Nucl. Eng. Des. 238 (3), 566-576.

Kuzmin, D., Turek, S., 2004. High-resolution FEM-TVD schemes based on a fully multidimensional flux limit. J. Comput. Phys. 198, 131-158.

Launder, B.E., Reece, G.J., Rodi, W., 1975. Progress in the development of a Reynoldsstress turbulence closure. J. Fluid Mech. 68, 537-566.

Mahaffy, J., Chung, B., Song, C., Dubois, F., Graffard, E., Ducros, F., Heitsch, M., Scheuerer, M., Henriksson, M., Komen, E., Moretti, F., Morii, T., Muehlbauer, P., Rohde, U., Smith, B. L., Watanabe, T., Zigh, G., 2007. Best Practice Guidelines for the use of CFD in Nuclear Reactor Safety Applications. NEA/CSNI/R(2007)5.

Nicoud, F., Ducros, F., 1999. Subgrid-scale stress modelling based on the square of the velocity gradient tensor. Flow Turbul. Combust. 62, 183-200.
Prasser, H.-M., Böttger, A., Zschau, J., 1998. A new electrode-mesh tomograph for gasliquid flows. Flow Meas. Instrum. 9, 111-119.

Prasser, H.-M., Grunwald, G., Höhne, T., Kliem, S., Rohde, U., Weiss, F.-P., 2003. Coolant mixing in a PWR - deboration transients, steam line breaks and emergency core cooling injection - experiments and analyses. Nucl. Technol. 143 (1), 37-56.

Reichardt, H., 1951. Vollständige Darstellung der turbulenten

Geschwindigkeitsverteilung in glatten Leitungen. Zeitschrift für Angewandte Mathematik und Mechanik 31, 208.

Rohde, U., Kliem, S., Höhne, T., Karlsson, R., Hemström, B., Lillington, J., Toppila, T. Elter, J., Bezrukov, Y., 2005. Fluid mixing and flow distribution in the reactor circuit: Measurement data base. Nucl. Eng. Des. 235, 421-443. 